\section{APPENDIX A: INDIVIDUAL ABUNDANCES}

In this Appendix, we present separately our detailed results for every element clearly identified in the spectrum of $\iota$ Her. The following sections are presented in ascending order of atomic number. The figures shows the observation in black (solid lines), the calculated model using the assigned abundance in blue (dash-dot-dot-dot lines) and calculated model in the absence of the specified element in red (long dash lines). In all figures, the vertical axis represents the normalized flux and the horizontal axis is the wavelength in Angstrom units, in vacuum.

\subsection{Boron, $\mathrm{Z}=5$}

There are only a few boron lines in our VALD line list. According to the Saha equation (see Table 3), the dominant states of ionization in the atmosphere are B II and III. We have used the B II resonance line at $1362.461 \AA$ (Ryabtsev, 2005) for abundance determination. This is the only boron line expected to be detectable in our spectrum, and fortunately it is not heavily blended. The oscilla" tor strength is very precisely known (it has quality "A" on the NIST Atomic Spectra Database scale, corresponding to an uncertainty of the transition probability of only about $3 \%$ (Kramida et al., 2014), and since this is a resonance line of a dominant ionization state, our LTE treatment should introduce no important errors. We estimate the uncertainty from fitting to be about \pm 0.1 . Therefore the abundance is $\log \left(n_{\mathrm{B}} / n_{\mathrm{H}}\right)=-10.1 \pm 0.1$ for this element. Figure 1 shows the fit to the observed profile.

Boron is a rare and fragile light element that is not produced through stellar nucleosynthesis (except for the very minor PP III chain), but is destroyed in the interiors of stars. There are several suggestion regarding the formation of this element, such as cosmic ray interactions with the ISM, or supernova neutrino spallation on particularly ${ }^{4} \mathrm{He}$ or ${ }^{12} \mathrm{C}$ (see Vangioni-Flam et al., 2000 for extensive details).

In comparison with the solar abundance of $\log \left(n_{\mathrm{B}} / n_{\mathrm{H}}\right)=$ $-9.30 \pm 0.03$ (Asplund et al., 2009), the abundance value found here is about a factor of 6 smaller. The presence of boron may indicate that outer layers of $\iota$ Her have at most been partially mixed into the stellar interior since the period of star formation. (In contrast, for instance, in Sirius A, the absence of detectable boron is consistent with the hypothesis that the outer layers were earlier inside its companion (Landstreet, 2011).)

\subsection{Carbon, $\mathrm{Z}=6$}

Carbon is a very abundant element in the spectrum of $\iota$ Herculis. In the VALD line list used here, most of the carbon lines are in neutral form. The rest are lines of C II, C III, and C IV. The distribution of carbon over ionization states in LTE in the atmosphere of $\iota$ Her is shown in Table 3. Most carbon is in the form of C II and C III, and both contribute numerous lines. However, even though it is a very minor constituent, weaker $\mathrm{C}$ I lines are evident throughout the spectrum. No C IV lines are detected.

We determined the abundance of carbon using a simultaneous fit to lines of C I UV multiplet (4) at $1329 \AA$, C II UV multiplet (11) at $1324 \AA$ and UV muliplet (1) at $1335 \AA$, the C III UV multiplet (4) between 1172 and $1180 \AA$, and UV multiplet (9) at $1247 \AA$. Although most of these lines have saturated line cores, several have strong wings that are reasonably sensitive to abundance. A good global fit is found for a logarithmic abundance of -3.55 , quite close to the solar value.

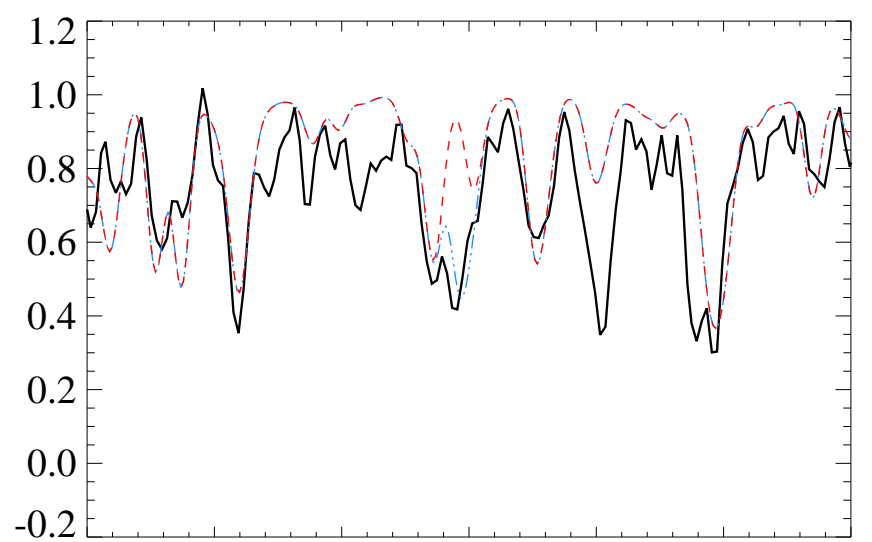

$\begin{array}{lllllll}1361.0 & 1361.5 & 1362.0 & 1362.5 & 1363.0 & 1363.5 & 1364.0\end{array}$

Figure 1. This figure shows the observation (in black), calculated model with $\log \left(n_{\mathrm{B}} / n_{\mathrm{H}}\right)=-10.1$ (in blue) and without boron (in red).

The $5 \mathrm{C}$ III triplet lines at 1174-76 $\AA$ and the singlet line at $1247.38 \AA$ have "A+" $g f$ values according to NIST. The ionisation energies for the $1175 \AA$ (Moore, 1970) triplets and the $1247 \AA$ line are 6.5 and $12.7 \mathrm{eV}$, respectively. The $4 \mathrm{C}$ II lines at $1323.9 \AA$ (Tachiev, 2000) have "A" $g f$ s, and are at $9.3 \mathrm{eV}$. The C II resonance lines at 1334-35 $\AA$ (Moore, 1970) also have "A" quality $g f$ s. NonLTE effects may play a role but the $g f$ values are very accurate, and contribute negligibly to the overall uncertainty.

It is notable that the worst fit in these windows is to the C I lines at $1329 \AA$. These lines are considered by Kramida et al. (2014) to have accuracy "B", with uncertainties of about \pm 0.04 in $\log g f$, so the atomic data make an unimportant contribution to the total error budget. The abundance derived from the best fit of these lines is about -4.65 , about one dex lower than that derived from lines of $\mathrm{C}$ II and C III, the two dominant ionization stages. This illustrates the dangers of relying on a (very) minor ionization stage, which in this case seems to be subject to quite significant overionisation.

It is worthwhile to mention that we removed from our VALD3 linelist, six lines of $\mathrm{C} I$ at the following wavelengths; 1253.410, $1253.467,1253.539,1253.541,1254.489,1254.511 \AA$, due to apparently inaccurate values of oscillator strength and/or damping constants. There are large inconsistencies between the values reported in VALD2 and VALD3, and neither set resulted in a good fit with our observation. Figure 2 shows the fit to the observed profile at these wavelengths.

\subsection{Nitrogen, $Z=7$}

The selected subset of the VALD database contains overall 233 lines of nitrogen. Almost half of these lines are for the neutral form (N I), and the rest are $\mathrm{N}$ II and N III, together with a few N IV lines. The strongest nitrogen contribution to the UV spectrum seems to be primarily due to N II. There are only a few strong lines of of $\mathrm{N} \mathrm{I}$ and N III. All the N IV lines are much weaker and less prominent. This distribution is consistent with the expected ionization ratios in the atmosphere according to the Saha equation (see Table 3).

With a few exceptions, most nitrogen lines are rather blended in the UV spectrum of this target. Although some N I lines appear largely unblended, the tiny fraction of $\mathrm{N}$ in this form makes abundance determination using such lines rather unreliable. Instead we have used the weakly blended N II line of UV multiplet (1) at $1083.99 \AA$ and lines of N II at 1275.04, 1276.20 and $1276.22 \AA$ 

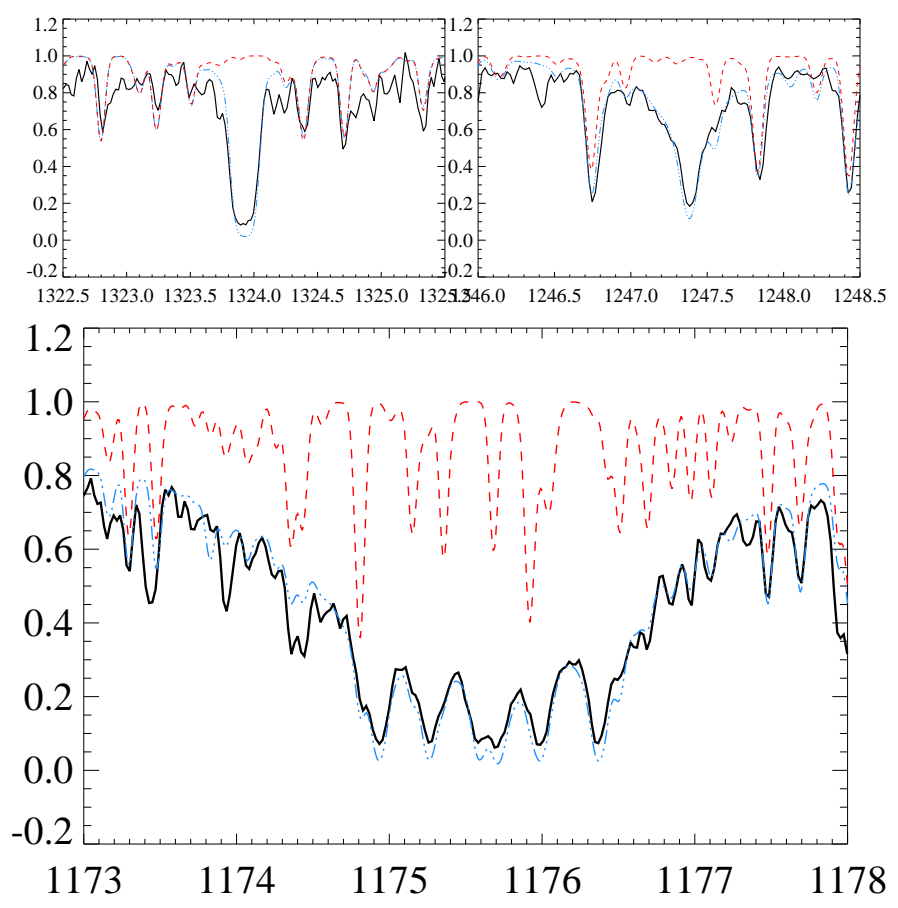

Figure 2. The figure shows the observation (in black), calculated model with $\log \left(n_{\mathrm{C}} / n_{\mathrm{H}}\right)=-3.55 \pm 0.30$ (in blue) and without carbon (in red). Top left: The unblended multiplet (11) triplet of $\mathrm{C}$ II at 1324 Å. Top right: The single line of multiplet (9) of C III at $1247 \AA$. Bottom: The C III lines of multiplet (4) around $1174 \AA$, with prominent wings extending to several $\AA$ from the deepest part of the feature.

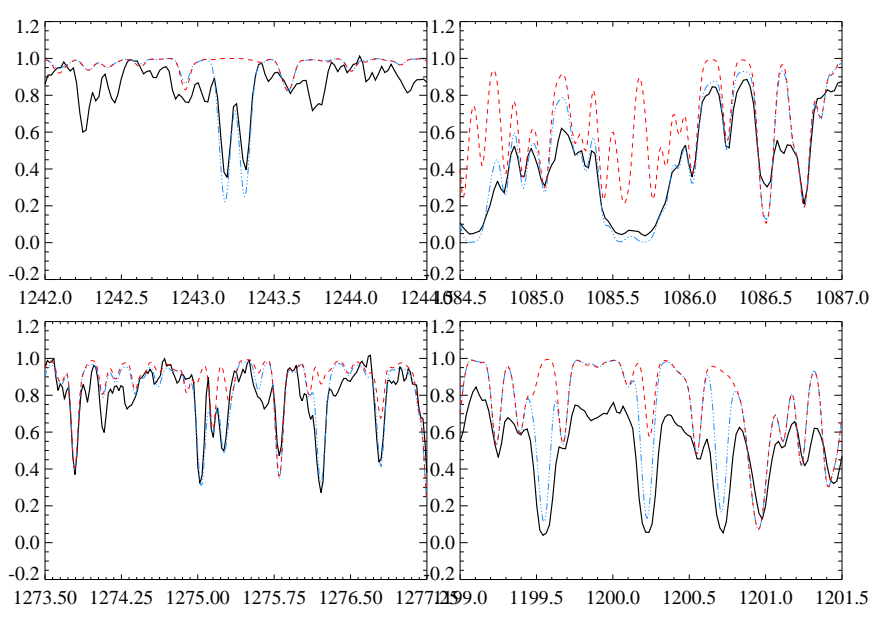

Figure 3. This figure shows the observation (in black), the best-fitting model calculated with $\log \left(n_{\mathrm{N}} / n_{\mathrm{H}}\right)=-4.65 \pm 0.80$ (in blue) and without nitrogen (in red). Top left: N I UV multiplet (5) at $1243 \AA$, Top right: N II multiplet (1) at $1085 \AA$, Bottom left: N II multiplet at 1275-1276 $\AA$, Bottom right: N I multiplet (1) at 1199-1200 ̊

to estimate the abundance of nitrogen. Uncertainty in both the zero point and continuum normalization, together with the fairly weak dependence of line strength on abundance, lead to a large uncertainty on the best compromise abundance of $-4.65 \pm 0.8$ dex.

Several observed lines as well as the model are shown in Figure 3

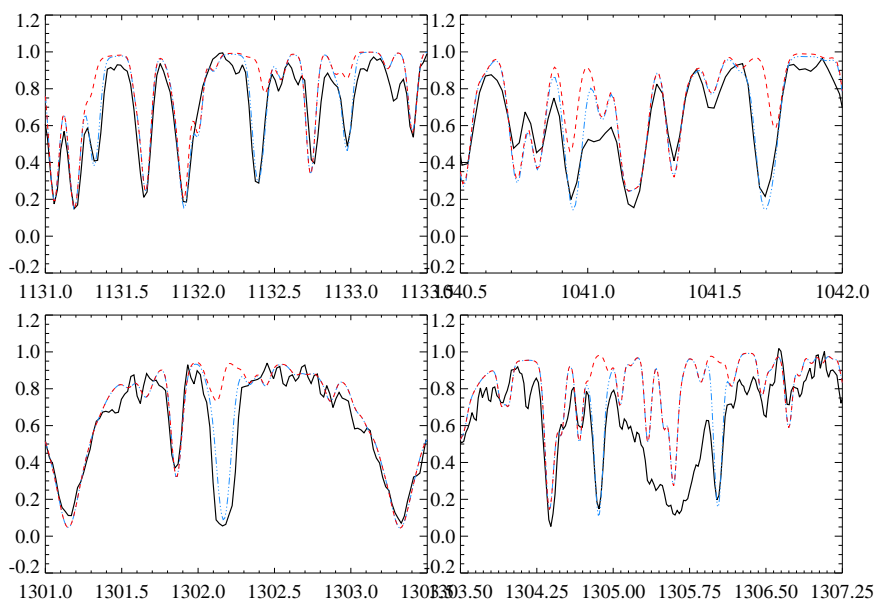

Figure 4. The figure shows the observed line (in black), the best-fitting model calculated with $\log \left(n_{\mathrm{O}} / n_{\mathrm{H}}\right)=-3.5 \pm 0.3$ (in blue) and without oxygen (in red). The lines are blended with iron. Top left: O II quartet lines at $1132 \AA$. Top right: O I triplet lines at $1041 \AA$, Bottom left: Strong O I at $1302 \AA$, Bottom Right: O I triplet lines at 1305-1306 ̊.

\subsection{Oxygen, $\mathrm{Z}=8$}

Oxygen- has a few very strong but blended lines in the spectrum of $\iota$ Herculis. The selection of VALD lines used here contains 218 oxygen lines, of which half are in neutral form and the rest are in the form of O II and O III, together with a few O IV lines. The UV spectrum of $\iota$ Herculis shows a strong presence of O II and $\mathrm{O}$ III with a moderate contribution from $\mathrm{O}$ I and absolutely no trace of OIV, which is expected given the effective temperature of this target. This distribution is consistent with the expected ionization ratios measured from the Saha equation (see Table 3).

We used the strong O II quartet lines at $1131 \AA$. These lines are slightly blended with nickel and iron, for which we have reasonable abundance estimates (see $1.17 \& 1.19$. The best-fitting model yields $\log \left(n_{\mathrm{O}} / n_{\mathrm{H}}\right)=-3.5 \pm 0.3$. We have used the strong O I UV multiplet (3) at 1039-1041 $\AA$ to confirm this value; Figure 4 shows only the longer wavelength end of the feature due to a long gap between strong lines. We also used the strong 1302-1306 $\AA$ lines of O I of UV multiplet (2); in Figure 4, we have shown only the short wavelength end. These lines are blended with iron lines (see 1.17). The best-fitting model results in the same value of $\log \left(n_{\mathrm{O}} / n_{\mathrm{H}}\right)=-$ $3.5 \pm 0.3$, thus we adopted this value for the oxygen abundance. Figure 4 shows the observation and the models.

\subsection{Magnesium, $\mathrm{Z}=12$}

In our subset of VALD database, there are only a few magnesium lines. The dominant ionization stage is predicted by the Saha equation to be Mg III (see Table 3). However, because the lowest excited states are more than $50 \mathrm{eV}$ above the ground state, no lines of $\mathrm{Mg}$ III are detectable in our window. We are therefore obliged to use lines of the weakly populated $\mathrm{Mg}$ II. There are numerous lines in our window that arise from low excitation levels about $4.5 \mathrm{eV}$ above ground, and even a couple of resonance lines. However, all these lines have very small $\log g f$ values, around -3 .

We used the Mg II doublet at $1367 \AA$ to determine the abundance of magnesium. We find $\log \left(n_{\mathrm{Mg}} / n_{\mathrm{H}}\right)=-3.90 \pm 0.19$. We confirmed this value using the strong line at Mg II $1240 \AA$. Figure 5 shows the fit to the observed profile at these wavelengths. 


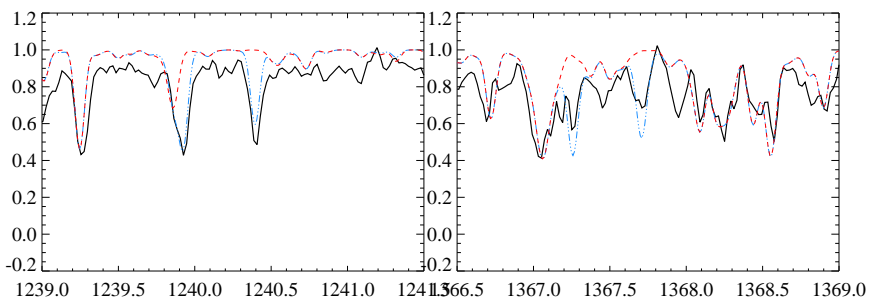

Figure 5. This figure shows the observation (in black), the calculated model with $\log \left(n_{\mathrm{Mg}} / n_{\mathrm{H}}\right)=-3.9 \pm 0.19$ (in blue) and without magnesium (in red). left: $\mathrm{Mg}$ II doublet at $1367 \AA$, right: strong line at Mg II $1240 \AA$

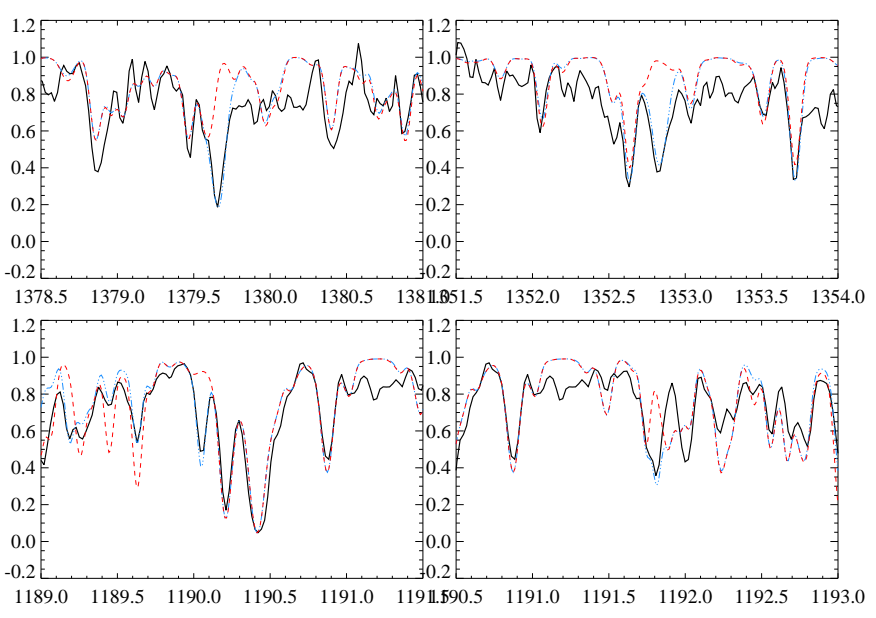

Figure 6. This figure shows the observation (in black), the calculated model with $\log \left(n_{\mathrm{Al}} / n_{\mathrm{H}}\right)=-5.90 \pm 0.58$ (in blue) and without aluminum (in red). Top left: $\mathrm{Al}$ III line at $1379 \AA$. Top right: $\mathrm{Al}$ III doublet line at $1352 \AA$, Bottom left: Al II triplet line at $1189 \AA$, Bottom right: $\mathrm{Al}$ II triplet line at $1191 \AA$.

\subsection{Aluminum, $Z=13$}

Aluminum is most prominent at longer wavelengths. Our selection of the VALD lines contains 88 aluminum lines in total; more than half of them are in the form of Al II and the rest are in Al III. At this temperature, almost all of aluminum is expected to be in Al III with a moderate $\mathrm{Al}$ IV contribution (see table 3 ).

In the spectrum of $\iota$ Herculis, most of the aluminum lines present are contributed by $\mathrm{Al}$ II, with a moderate number of $\mathrm{Al}$ III lines and no trace of Al IV. The lack of lines of Al IV is clearly due to the atomic energy structure, which has no excited states below $75 \mathrm{eV}$.

The available lines of $\mathrm{Al}$ are heavily blended throughout the spectrum. We used the Al III line at $1379 \AA$ which is only blended with iron (see 1.17 . The best-fitting model results in the abundance of $\log \left(n_{\mathrm{Al}} / n_{\mathrm{H}}\right)=-5.90 \pm 0.58$ for aluminum. We confirmed this value by fitting the following lines; Al II triplet lines at 1189$1190 \AA$, a strong Al III doublet line at $1352 \AA$, and another Al II triplet line at $1191 \AA$. Figure 6 shows the observation and the model fits.

\subsection{Silicon, $\mathrm{Z}=14$}

The VALD lines selected here include 361 silicon lines. The majority of these lines are in the form of Si II and Si III and a small fraction of them are in the form of Si I and Si IV. In the UV spectrum of $\iota$ Herculis, silicon is largely observed in the form of Si II and Si III with a very minor contribution from Si IV and no trace
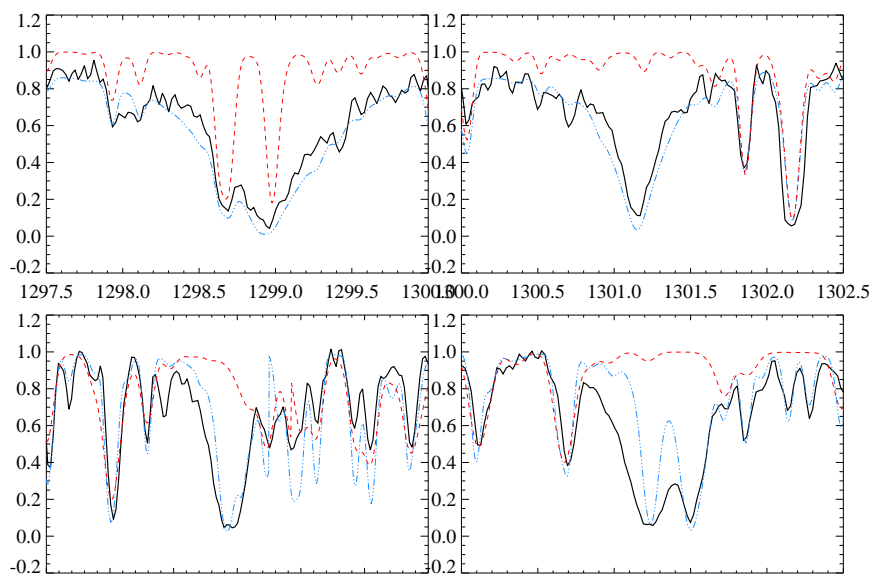

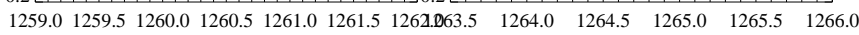

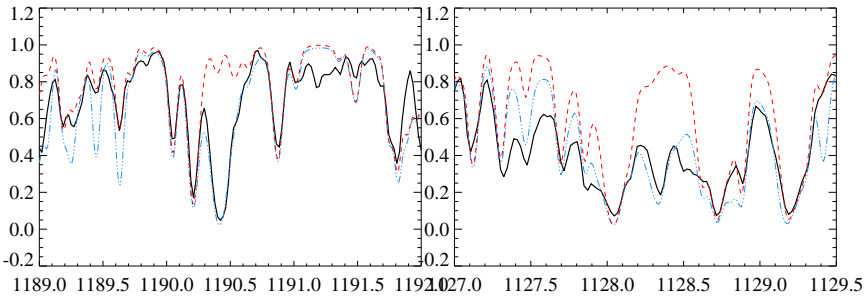

Figure 7. This figure shows the observation (in black), the best-fitting model spectrum with $\log \left(n_{\mathrm{Si}} / n_{\mathrm{H}}\right)=-4.65 \pm 0.26$ (in blue) and without silicon (in red). Top: Strong Si III lines of multiplet (4) between 1294$1310 \AA$, we only show $1298 \AA$ (left) and $1301 \AA$ (right), Middle: Multiplet (5) of Si II between 1260-64 $\AA$ around $1260 \AA$ (left) and $1264 \AA$ (right), Bottom left: Si II multiplet (4) between 1190-93 Å, Bottom right: A strong Si,IV line at $1128 \AA$.

of Si I. This is generally consistent with the expectations from the Saha equation. The fact that lines of Si II, Si III and Si IV are all found in the spectrum is because all these ionization stages have lines arising from the ground state or low lying levels that occur in the window we are modeling.

We determined the abundance of Si using the relatively unblended and strong Si III lines of multiplet (4) between 1294$1310 \AA$, and the lines of multiplet (5) between 1108-1114 $\AA$. We find the abundance of $\log \left(n_{\mathrm{Si}} / n_{\mathrm{H}}\right)=-4.65 \pm 0.26$ for silicon. For confirmation, we have used this abundance to model the blended multiplet (4) and (5) lines of Si II at 1190-93 $\AA$ and 1260-64 $\AA$, and a strong Si IV multiplet (3) line at $1128 \AA$. Figure 7 shows the best-fitting models and the observed spectrum.

Note that the fit to the lines of Si II in the middle panel is poor, and suggests an abundance of Si larger than we have chosen. Major discrepancies between abundances deduced with Si II and Si III are common in hot stars, and may represent important departures from LTE or possibly stratification of Si in the atmosphere (Bailey \& Landstreet, 2013), but in this case the discrepancies may be due to unidentified blends.

\subsection{Phosphorus, $Z=15$}

There are overall 133 phosphorus lines in the subset of the VALD database selected here; mostly in the form of P II, a smaller subset are in the form of PIV, P III, and there are only a few PI and $\mathrm{P} V$ lines. In the UV spectrum of $\iota$ Herculis, phosphorus lines are mostly observed in the form of P II and P III and there is a very minor contribution from other ionization states (P IV, P V and P I). 


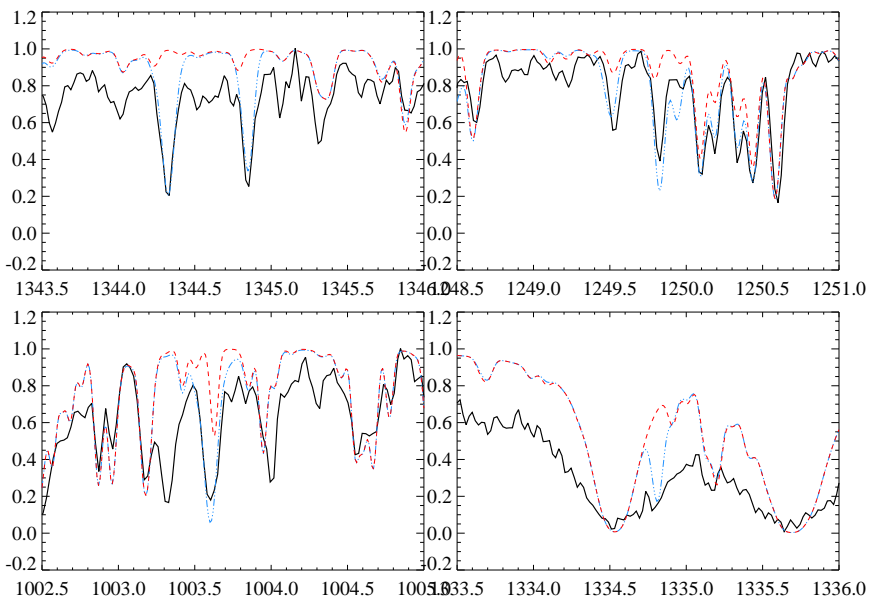

Figure 8. This figure shows the observation (in black), the calculated model with $\log \left(n_{\mathrm{P}} / n_{\mathrm{H}}\right)=-6.7 \pm 0.7$ (in blue), and without phosphorus (in red). Top left: A P III doublet at $1344 \AA$, Top right: A P II line at $1249 \AA$, Bottom left: A P III at $1003 \AA$ Bottom right: A resonance P III line at $1334 \AA$.

This is consistent with the ionization ratios obtained from the Saha equation at this temperature (see Table 3). All the P II and P III lines in the wavelength range studied here arise from low lying energy levels $(0-1.1 \mathrm{eV})$ which explains their clear appearance in the observed spectrum.

We have used a strong P III tripet of UV multiplet (1) at 1344$45 \AA$. These lines both arise from low initial energy level $(0.07 \mathrm{eV})$ with a fairly accurate (Kramida et al., 2014) oscillator strength value of "D" $\log g f \sim-1.5$. They are slightly blended with iron for which we have a reasonable abundance determination (see 1.17 . Modeling these lines yields $\log \left(n_{\mathrm{P}} / n_{\mathrm{H}}\right)=-6.7 \pm 0.7$. We confirmed this value using two strong lines of P II at $1249.8 \AA$ and P III at $1003.6 \AA$ with $\log g f$ values close to zero and a P III resonance line at $1334.8 \AA$ with " $D$ " $\log g f$ values close to -1 . Figure 8 shows the observation and the best-fitting models.

\subsection{Sulfur, $\mathrm{Z}=16$}

There are 474 lines in the subset of the VALD database used here. In the observed UV spectrum of $\iota$ Herculis as well as the VALD database, sulfur has a few strong lines in the form of S II, S III and $\mathrm{S}$ IV and a large number of weaker S I lines. This is consistent with the expected ionization ratios predicted by the Saha equation.

We have modeled the strong S III resonance lines of UV multiplet (1) at $1200 \AA$. The best-fitting model results in the value of $\log \left(n_{\mathrm{S}} / n_{\mathrm{H}}\right)=-5.20 \pm 0.10$. We confirmed this value using a line of S IV multiplet (1) at $1072 \AA$. This line is blended with iron, nickel and manganese, but can still be used for our purposes (see 1.17 1.19 1.16. We also used S II resonance lines of multiplet (1) in the window between $1250-59 \AA$, and a S II resonance line at $1072 \AA$. The oscillator strength of all the lines used here are around -1 with a "D" accuracy (Kramida et al., 2014) and they all arise from low energy levels. The observation and models are shown in Figure 9

\subsection{Chlorine, $\mathrm{Z}=17$}

Chlorine has 117 lines in our selected database. The majority of them are in neutral form. In our spectral window of $\iota$ Herculis, chlorine is observed mostly in shorter wavelengths. The observed spectrum is mostly dominated by $\mathrm{Cl}$ I lines with minor contribution
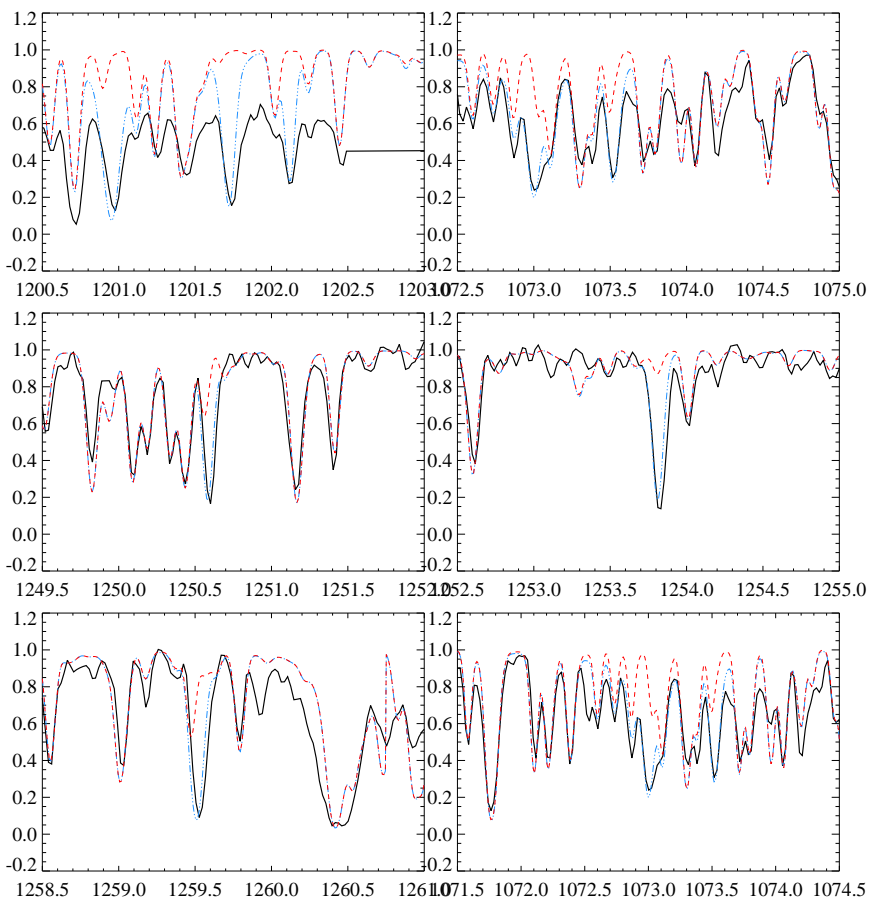

Figure 9. This figure shows the observation (in black), the calculated model with $\log \left(n_{\mathrm{S}} / n_{\mathrm{H}}\right)=-5.20 \pm 0.10$ (in blue), and without sulfur (in red). Top left: The strong S III triplet lines at $1200 \AA$, Top right: The S IV doublet at $1072 \AA$, Middle: A strong S II resonance doublet at $1250 \AA$, Bottom left: A strong S II resonance line at $1259 \AA$, Bottom right: A strong S II line at $1072 \AA$

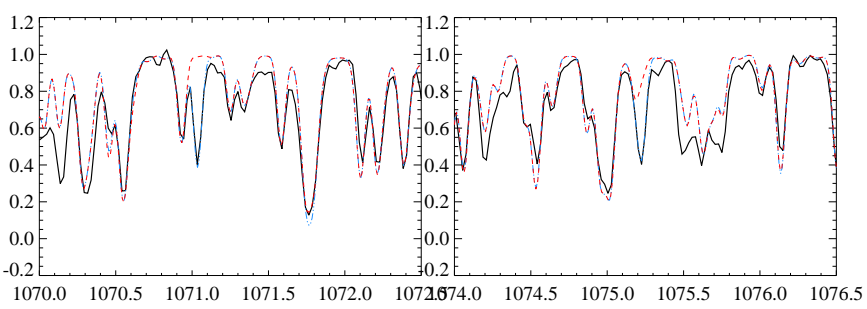

Figure 10. This figure shows the observation (in black), the calculated model with $\log \left(n_{\mathrm{Cl}} / n_{\mathrm{H}}\right)=-7.150 \pm 0.100$ (in blue), and without chlorine (in red). Left: $\mathrm{A} \mathrm{Cl} \mathrm{II}$ line at $1071 \AA$, Right: $\mathrm{A} \mathrm{Cl} \mathrm{II} \mathrm{line} \mathrm{at} 1075 \AA$.

from other ionization states ( $\mathrm{Cl} \mathrm{II}, \mathrm{Cl} \mathrm{III}$, and $\mathrm{Cl} \mathrm{IV}$ ). As predicted by the Saha equation (see Table 3), the dominant ionization state at this temperature should be $\mathrm{Cl}$ III. However, this ionization state contributes only very weakly. The lowest excited states of Cl III are $18 \mathrm{eV}$ above the ground state (Kramida et al., 2014), which explains why there are virtually no $\mathrm{Cl}$ III lines seen in the spectral window studied here.

We determined the abundance of chlorine using the unblended Cl II resonance line of multiplet (1) at $1071 \AA$ shown in Figure 10 This line has a "C" $\log g f$ value of roughly -1 . The bestfitting model to this line results in the value of $\log \left(n_{\mathrm{Cl}} / n_{\mathrm{H}}\right)=-$ $7.15 \pm 0.10$. We confirmed this value by also modeling the $\mathrm{Cl}$ II line at $1075 \AA$. This line also arises from a very low energy level of $0.1 \mathrm{eV}$ and has a "C" $\log g f$ of -1.7 (Kramida et al., 2014). The observation and models are shown in Figure 10 


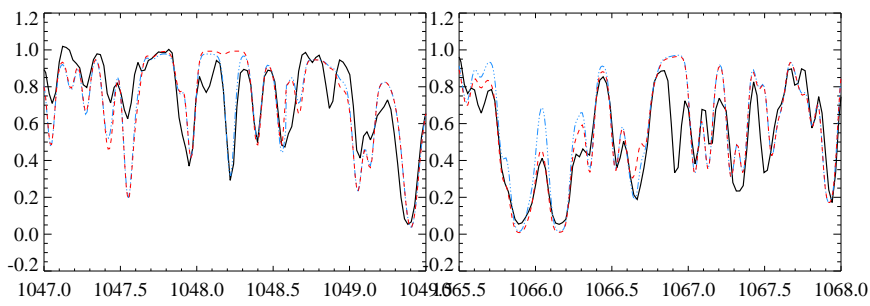

Figure 11. This figure shows the observation (in black), the calculated model with $\log \left(n_{\mathrm{Ar}} / n_{\mathrm{H}}\right)=-4.900 \pm 0.100$ (in blue), and without argon (in red). Left: Ar I line at $1048 \AA$ Right: Ar I line at $1066 \AA$.

\subsection{Argon, $Z=18$}

Argon appears with 66 lines in the subset of VALD database selected here. In the spectrum of $\iota$ Herculis, argon is observed mostly in the form of weak Ar II lines with a minor contribution from stronger Ar I and Ar III lines. As measured from the Saha equation, the dominant ionization state at this temperature is Ar II (see Table 3). The reason for the under-population of Ar II lines in the observed spectrum is that in this wavelength window, almost all of the Ar II lines arise from high excitation energy levels which are at least $\sim 16 \mathrm{eV}$ above the ground state.

For the abundance determination, we modeled two suitable lines of Ar I; first a UV multiplet (1) line at $1048 \AA$ as well as another UV multiplet (1) line at $1066 \AA$. These two lines are both resonance lines and have relatively well determined " $\mathrm{C}+$ " $\log g f$ values of around -0.5 and -1.0 , respectively (Kramida et al., 2014), and the line at $1048 \AA$ appears unblended. The best-fitting model yields $\log \left(n_{\mathrm{Ar}} / n_{\mathrm{H}}\right)=-4.90 \pm 0.10$ in both cases. Figure 11 shows the observation and the models.

\subsection{Calcium, $Z=20$}

In the UV spectrum of $\iota$ Herculis, calcium is not strongly observed. The subset of the VALD database selected here contains overall 43 calcium lines which are almost equally distributed between Ca II and Ca III. The prediction of the Saha equation shows that Ca III must be the dominant state of ionization at this temperature (see Table 3). However, because the lowest excited states are more than $30 \mathrm{eV}$ above ground, there is almost no contribution from the Ca III state in the observed spectrum of $\iota$ Herculis.

Despite the lack of clean and unblended lines, we could determine the abundance using blended lines. Modeling the two Ca II lines in the 1369 and $1432 \AA$ windows yields $\log \left(n_{\mathrm{Ca}} / n_{\mathrm{H}}\right)=-$ $5.30 \pm 0.10$. These lines are suited for our purpose since they both arise from a low energy state $(\sim 1.6 \mathrm{eV})$. They both have relatively well determined "C" $\log g f$ values of -0.8 (Kramida et al., 2014). Figure 12 shows the observations and models.

\subsection{Titanium, $\mathrm{Z}=\mathbf{2 2}$}

Titanium has 156 titanium lines in our database. Most of them are in the form of Ti III, and a smaller fraction of them are in Ti II with little to contribution from Ti IV. In the UV spectrum of $\iota$ Herculis, Ti III is dominantly observed and there is a minor contribution from Ti II lines at longer wavelengths. This is consistent with the ionization ratios calculated from the Saha equation for this temperature (see Table 3).

We determined the abundance of titanium using lines of the two strong Ti III multiplets (1) and (2) in the 1286-93 $\AA$ window.

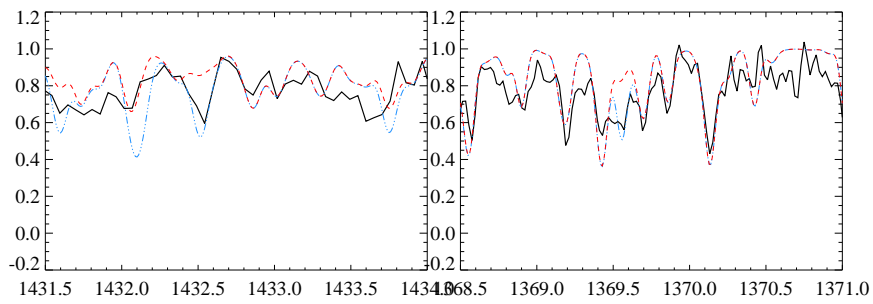

Figure 12. This figure shows the observation (in black), the calculated model using $\log \left(n_{\mathrm{Ca}} / n_{\mathrm{H}}\right)=-5.30 \pm 0.10$ (in blue), and without calcium (in red). Left: Ca II lines at $1432 \AA$, Right: Ca II line at $1369 \AA$.

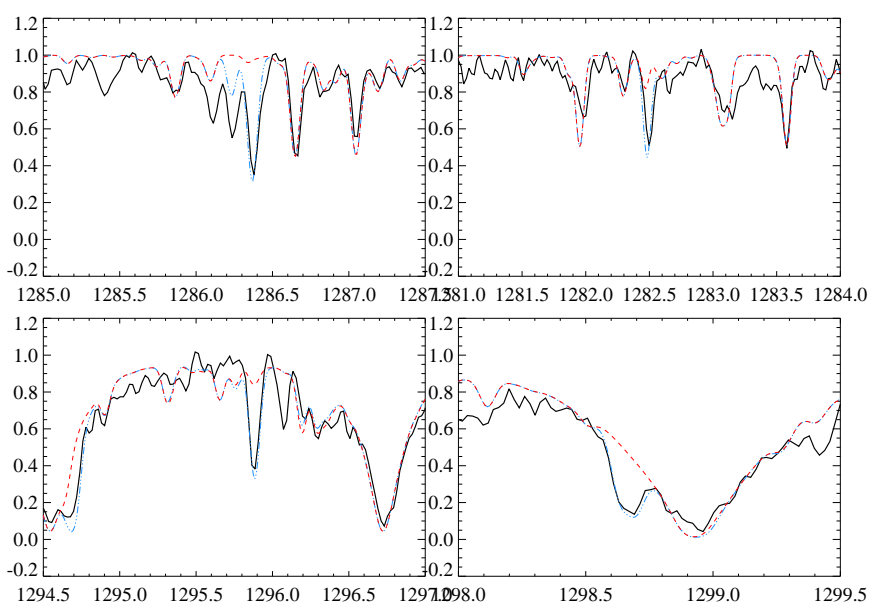

Figure 13. This figure shows the observation (in black), the calculated model with $\log \left(n_{\mathrm{Ti}} / n_{\mathrm{H}}\right)=-6.90 \pm 0.17$ (in blue), and without titanium (in red). All the plots belong to the same ionization state Ti III; Top left: A resonance line at $1286 \AA$, Top right: at $1282 \AA$, Bottom left: at $1295 \AA$, Bottom right: at $1298 \AA$

They are suitable choices because they arise from low lying energy levels ( 0.00-0.05) and have relatively well determined ("D") $\log g f$ values of around -0.5 . Only parts of these multiplets are shown in Figure 13

The best-fitting model results in the abundance $\log \left(n_{\mathrm{Ti}} / n_{\mathrm{H}}\right)=-6.90 \pm 0.17$. We confirmed this abundance by modeling the Ti III UV resonance multiplet (2) at $1282 \AA$ and Ti III resonance lines at 1295-98 $\AA$. These lines all have a "D" $\log g f$ values of around -0.50 and despite a slight blending with nickel (see 1.19 ) they still serve our purpose. Figure 13 shows the observed lines as well as the calculated models.

\subsection{Vanadium, $\mathrm{Z}=\mathbf{2 3}$}

Our line database contains 234 vanadium lines. All are in the form of V III. In the spectrum of $\iota$ Herculis vanadium is also observed only as V III. The predictions of the Saha equation shows (see Table 3) that V III is the dominant state of ionization and V I, V II are severely underpopulated in this temperature and wavelength range. The lines of V IV are absent because the allowed transitions in our spectral region arise from excited states that are higher than $\sim 18 \mathrm{eV}$.

We have used the four strong and fairly clean V III lines at $1154-1160,1252,1332 \AA$. They all arise from low lying energy lev- 

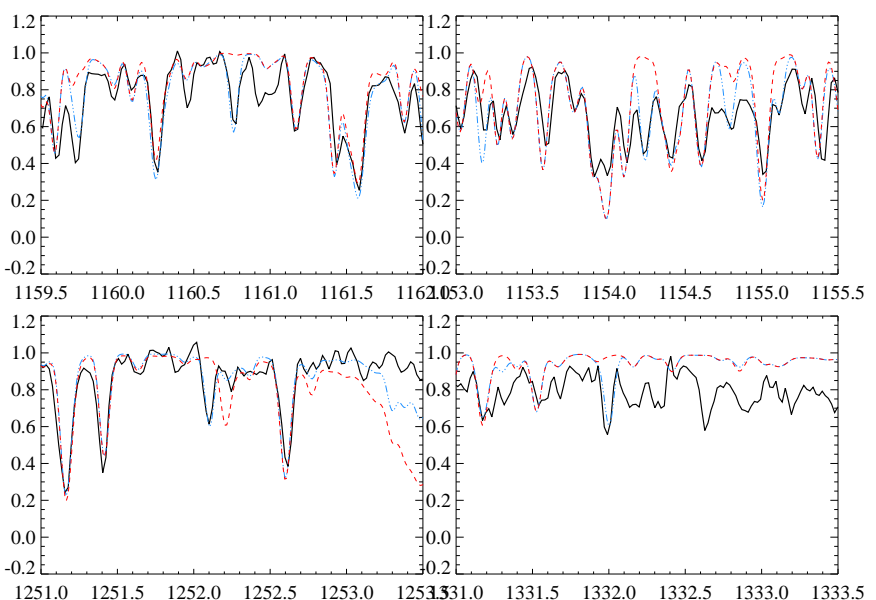

Figure 14. This figure shows the observation (in black), the calculated model with $\log \left(n_{\mathrm{V}} / n_{\mathrm{H}}\right)=-8.15 \pm 0.18$ (in blue), and without vanadium (in red). All of the plots belong to the same state of ionization; V III. Top left: $1160 \AA$, Top right: $1154 \AA$, Bottom left: $1252 \AA$, Bottom right: $1332 \AA$

els $(\sim 1.5 \mathrm{eV})$ and $\log g f$ values around zero. Despite slight blending with Ni II line (see 1.19 , they still serve our purpose.

The best-fitting model to these lines results from a vanadium abundance of $\log \left(n_{\mathrm{V}} / n_{\mathrm{H}}\right)=-8.15 \pm 0.18$. Figure 14 shows the best-fitting model to the observation. The blending of nickel and vanadium provides a reasonable fit to the observation.

\subsection{Chromium, $\mathrm{Z}=24$}

The selected line-list contains 1645 chromium lines. In the UV spectrum of $\iota$ Herculis, chromium is mostly observed as Cr III, with smaller contributions from $\mathrm{Cr}$ II and Cr IV. This is consistent with the ionization ratios predicted from the Saha equation (see Table 3.

We used the CrIII triplet line in the $1098 \AA$ window to determine the abundance. This line is unblended and it arises from a fairly low lying energy level of $2 \mathrm{eV}$ and loggf of $\sim-2$. The accuracy on the oscillator strengths of chromium lines are not known very well. The best-fitting model results in $\log \left(n_{\mathrm{Cr}} / n_{\mathrm{H}}\right)=-$ $6.10 \pm 0.65$.

We confirmed this value using Cr III triplet lines at $1040 \AA$ and $1064 \AA$ windows and a strong Cr III line at $1051 \AA$. What they all have in common is that they arise from low energy levels and have oscillator strengths that is between 0 and -1 . Figure 15 shows the observation and the best-fitting models.

\subsection{Manganese, $\mathrm{Z}=\mathbf{2 5}$}

In the database selected here, there are 1448 manganese lines. Almost all of them are in the form of Mn III, and the rest are in the form of Mn II, with very few lines of Mn IV. In the UV spectrum of $\iota$ Herculis manganese is observed mostly in the form of Mn III lines throughout, with a few strong Mn II lines. The contribution from Mn IV is too weak to be discernible. The measurements from the Saha equation, indicates that Mn III is the dominant state of ionization at this temperature which is consistent with our observation.

We have used the unblended strong Mn III lines in the $1088 \AA$ window to determine the abundance. We find $\log \left(n_{\mathrm{Mn}} / n_{\mathrm{H}}\right)=$ $6.78 \pm 0.45$ for manganese. We confirmed this value by modeling the Mn III doublet lines in the 1046, 1239 and $1111 \AA$ windows. All the lines chosen here have $\log g f$ values around -1 and arise

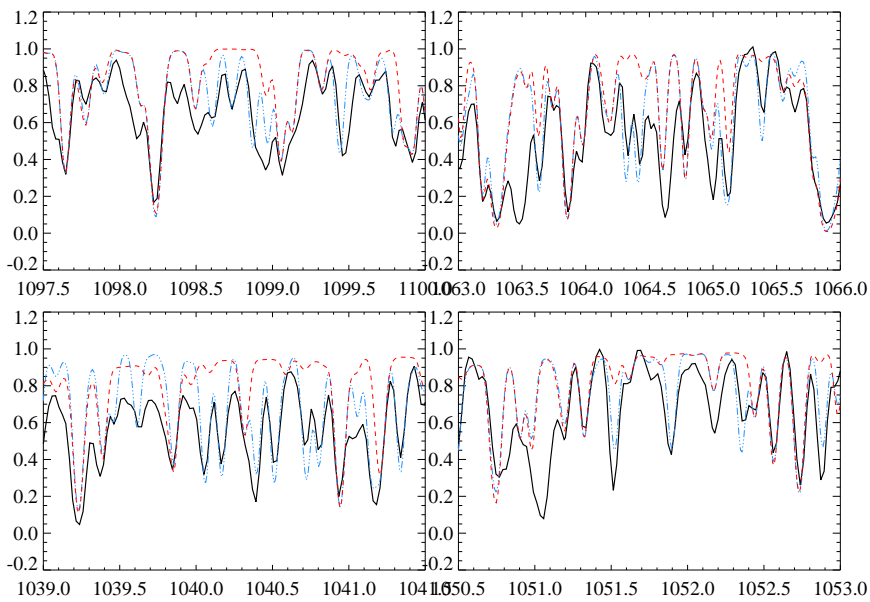

Figure 15. This figure shows the observation (in black), the calculated model with $\log \left(n_{\mathrm{Cr}} / n_{\mathrm{H}}\right)=-6.10 \pm 0.65$, and without chromium (in red). The plots belong to the same ionization state; Cr III. Triplet lines at $1098 \AA$ (Top left), Cr III triplet lines at $1064 \AA$ (Top right),Cr III resonance line with contributions from another multiplet at $1040 \AA$ (Bottom left), strong Cr III line at $1051 \AA$ (Bottom right)

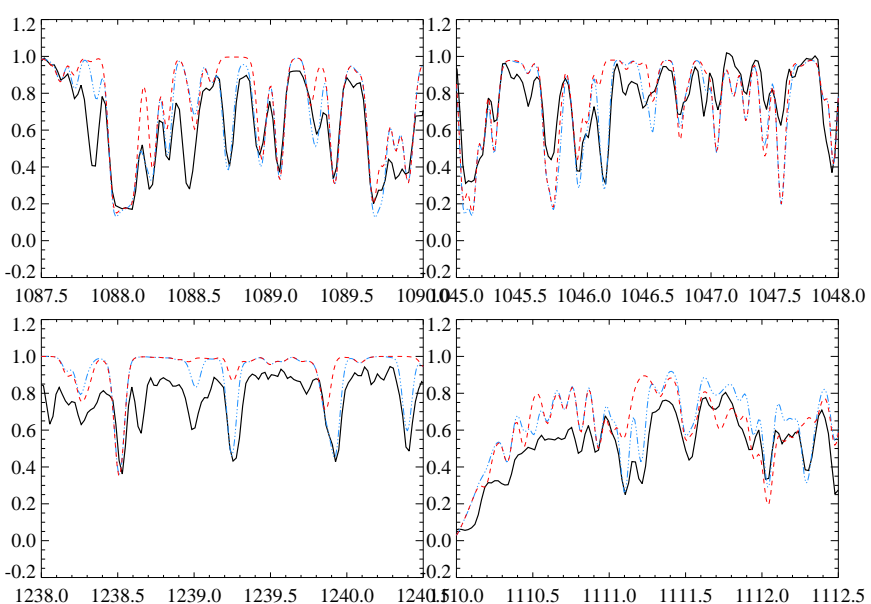

Figure 16. This figure shows the observation (in black), the calculated model with $\log \left(n_{\mathrm{Mn}} / n_{\mathrm{H}}\right)=-6.78 \pm 0.45$, and without manganese (in red). These plots belong to the same ionization state of Mn III; doublet lines at $1088 \AA$ (Top left), doublet lines at $1046 \AA$ (Top right), doublet lines at $1239 \AA$ (Bottom left), doublet lines at $1111 \AA$ (Bottom right).

from low lying energy levels $(\sim 4 \mathrm{eV})$. The observed spectrum and models are shown in Figure 16

\subsection{Iron, $\mathrm{Z}=26$}

Iron lines are prominent in almost every region of the UV spectrum of $\iota$ Herculis. Our selection of the VALD database contains 8165 iron lines. More than half of them are in the form of Fe II. The Fe III lines, even though fewer in number, seem to be stronger and deeper than the lines of Fe IV which are very weak.

In the UV spectrum of $\iota$ Herculis the majority of iron lines are observed in the form of moderately strong Fe II lines and fewer Fe III lines. Because the lowest excited state is $\sim 19 \mathrm{eV}$ above the ground state, Fe IV does not appear in in our observed spectral window. The calculations from the Saha equation (see Table 3) indicate that the dominant ionization state at this temperature is Fe III. Nev- 


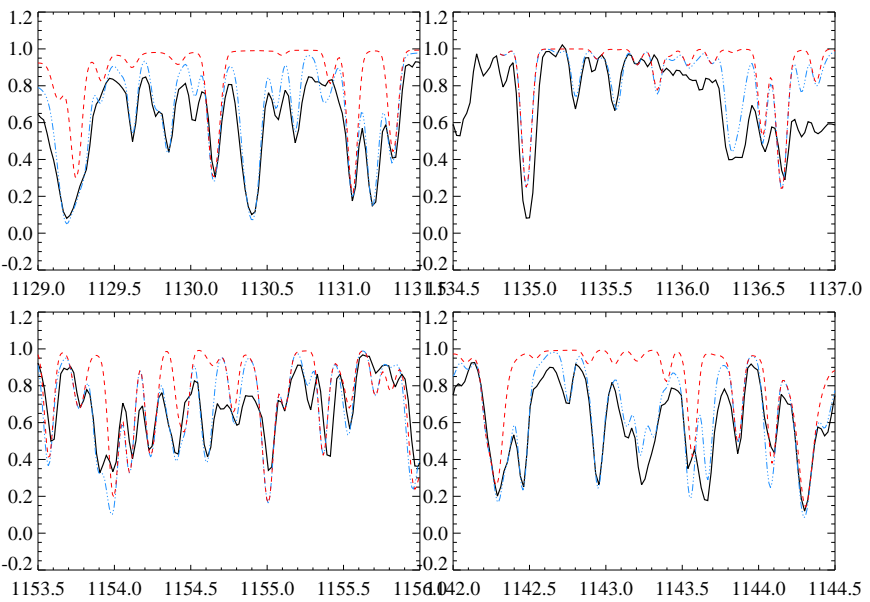

Figure 17. This figure shows the observation (in black), the calculated model with $\log \left(n_{\mathrm{Fe}} / n_{\mathrm{H}}\right)=-4.9 \pm 0.4$ (in blue), and without iron (in red). Top left: The Fe III lines at $1130 \AA$, blended with Fe II lines. Top right: Fe II doublets at $1135 \AA$. Bottom left: Fe III doublet at $1154 \AA$, Bottom right: The strong Fe III line at $1142 \AA$ blended with Fe II lines.

ertheless, Fe III lines do not appear strongly since for most such lines the lower level is on average $10 \mathrm{eV}$ or more above the ground state. In contrast the lower levels of strong Fe II lines are only slightly (about $4 \mathrm{eV}$ ) above the ground state which strengthens lines from this state.

Despite the frequency of Fe II lines in the spectrum, they are heavily blended and are not ideal for abundance measurements. We instead used the strong pair of Fe III lines in the $1130 \AA$ window with a slight overlapping blend from Fe II lines. This set of lines is suited for our purpose because they, not only are unblended, but also arise from very low lying energy state $(0.1 \mathrm{eV})$ with $\log g f$ of -1 (VALD3). The best-fitting model results in $\log \left(n_{\mathrm{Fe}} / n_{\mathrm{H}}\right)=-$ $4.9 \pm 0.4$.

We confirmed this value through modeling a cluster of iron lines in the 1142-43 $\AA$ window including Fe III and Fe II lines, Fe II lines around $1135-26 \AA$, and Fe III lines in the $1154 \AA$ window. All these lines arise from very low lying energy levels $(0-4 \mathrm{eV})$ and have $\log g f$ values around zero or -1 . Figure 17 shows the observation and the modeled spectra.

\subsection{Cobalt, $\mathrm{Z}=27$}

The selected database here contains 581 cobalt lines. In the UV spectrum of $\iota$ Herculis, cobalt is observed over the entire wavelength range. Almost all of the strong lines are in the form of Co III, with a few Co II lines and only one Co IV line. The Saha equation predictions shown in Table 3 are consistent with the observed ionization ratios.

We modeled a group of Co III in the window between 1043-88 and found $\log \left(n_{\mathrm{Co}} / n_{\mathrm{H}}\right)=-7.55 \pm 0.49$. These lines all arise from low energy states at $1.9 \mathrm{eV}$ with well determined oscillator strength values around -1 (VALD3). We tested this value by also modeling the resonance Co II line at $1466 \AA$. Figure 18 shows the best-fitting model and the observation.

\subsection{Nickel, $Z=28$}

The line list selected for this work contains 2145 nickel lines. More than half of the lines in the list are in the form of Ni III. A smaller

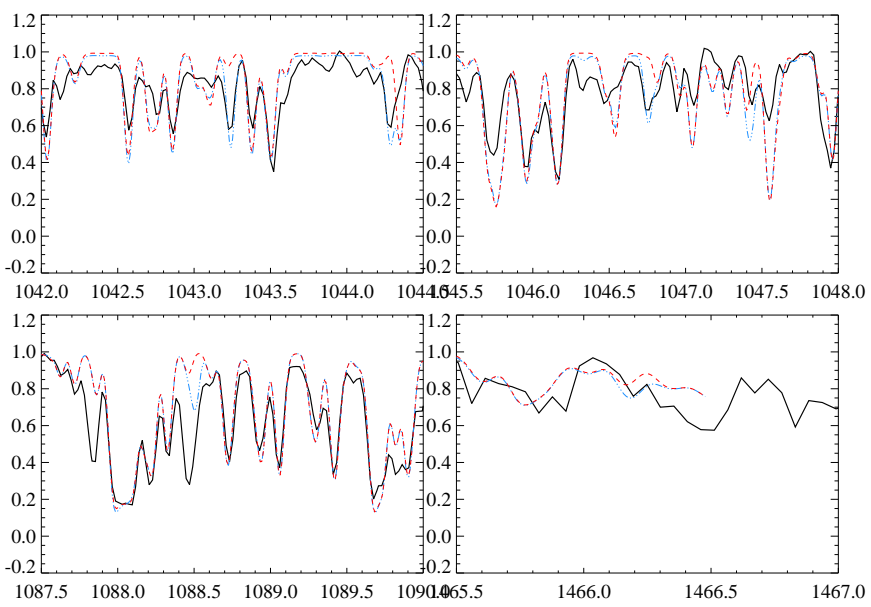

Figure 18. This figure shows the observation (in black), the calculated model with $\log \left(n_{\mathrm{Co}} / n_{\mathrm{H}}\right)=-7.55 \pm 0.49$ (in blue), and without cobalt (in red). Top left: Co III line at $1043 \AA$, Top right: Co III line at $1046 \AA$, Bottom left: Co III line at $1088 \AA$, Bottom right: Co II line at $1466 \AA$.

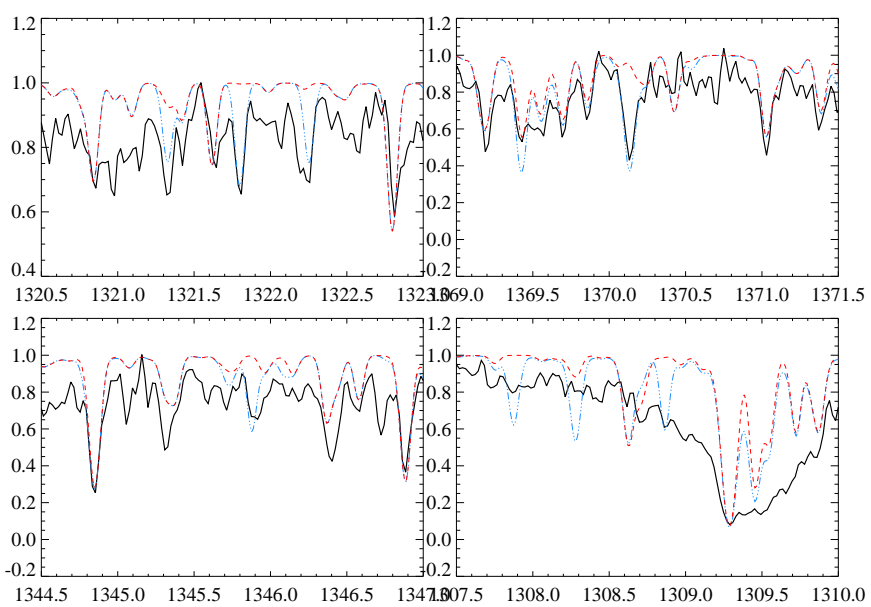

Figure 19. This figure shows the observation (in black), the calculated model with $\log \left(n_{\mathrm{Ni}} / n_{\mathrm{H}}\right)=-5.70 \pm 0.35$ (in blue), and without nickel (in red). Top left: Ni III triplet lines at 1321-22 $\AA$, Top right: a resonance Ni II lines at $1370 \AA$, Bottom left: a resonance Ni II line at $1345 \AA$, Bottom right: a resonance Ni II line at $1308 \AA$.

fraction is in the form of Ni II, with only a few lines of Ni IV. In the UV spectrum of $\iota$ Herculis nickel is observed mostly as Ni III and Ni II with very little contribution from Ni IV. The Ni IV lines hardly appear in the spectrum because the excited states are at least $14 \mathrm{eV}$ above the ground state (VALD3). This distribution is consistent with the ratios estimated from the Saha equation (see Table 3).

We determined the abundance of this element using the Ni III triplet lines in the 1321-22 $\AA$ window and found $\log \left(n_{\mathrm{Ni}} / n_{\mathrm{H}}\right)=$ $5.70 \pm 0.35$. These lines arise from $\sim 6 \mathrm{eV}$ and have $\log g f$ around -2 , but they are suitable for this purpose because they are clean and unblended.

We confirmed this value by modeling three resonance Ni II lines in three wavelength windows including 1308, 1345, $1370 \AA$. Figure 19 shows the resulting model using this abundance and the fit to the observation. 


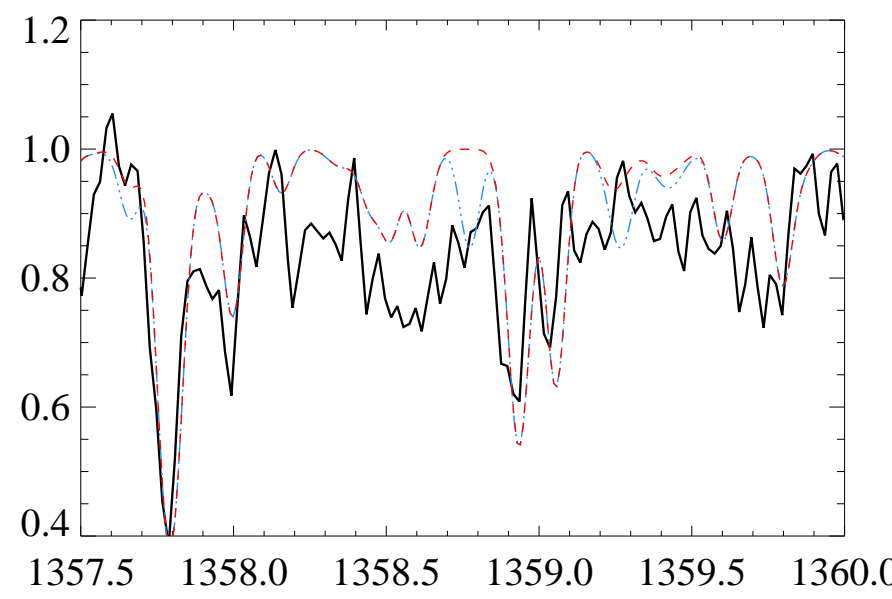

Figure 20. This figure shows the observation (in black), the calculated model with $\log \left(n_{\mathrm{Cu}} / n_{\mathrm{H}}\right)=-9.00$ (in blue), and without copper (in red). This is a resonance $\mathrm{Cu}$ II line at $1358 \AA$.

\subsection{Copper, $Z=29$}

In the subset of VALD database used here there are $62 \mathrm{Cu}$ II lines. In the UV spectrum of $\iota$ Herculis, copper is observed in the form of $\mathrm{Cu}$ II throughout the entire wavelength range studied here. The results of the Saha equation indicate that copper must be dominated by $\mathrm{Cu}$ III at this temperature which is inconsistent with our line list. The reason seems to be a lack of atomic data about $\mathrm{Cu}$ III.

We modeled the strong resonance line of $\mathrm{Cu}$ II at $1358 \AA$ (Ross, 1969) with $\log g f$ of almost 0 , and found an abundance of $\log \left(n_{\mathrm{Cu}} / n_{\mathrm{H}}\right)=-9.0$. This line is the only unblended line that we have available in our spectrum. We present this as an upper limit for this element due to lack of other unblended lines for comparison. The uncertainty could not be estimated on this value since there are not enough clean lines for comparison. The least uncertainty on this value is due to stellar parameter inaccuracy which is of the order of 0.04 dex (see $\$ 3.3$ ). Figure 20 shows the observation and the model.

\subsection{Zinc, $\mathrm{Z}=30$}

Zinc has 127 weak lines in the form of Zn III in our selected VALD line list. In the spectrum of $\iota$ Herculis, zinc is mostly observed in the longer wavelengths and it appears only in the form of $\mathrm{Zn}$ III. This ionization distribution is consistent with the ratios estimated from the Saha equation (see Table 3).

The lines are weak and heavily blended and arise from at least $9 \mathrm{eV}$ above the ground state. The lowest excited energy state belongs to a Zn III doublet line in the $1456-64 \AA$ window. These lines are weak but fairly unblended. We have used them for abundance determination and found $\log \left(n_{\mathrm{Zn}} / n_{\mathrm{H}}\right)=-6.85 \pm 0.20$. The uncertainty arises from the fact that each of these lines can best be modeled with a slightly different abundance (see Figure 21). The Zn III line in the $1359 \AA$ window can also be used to confirm this value. The observation and models are shown in Figure 21.

\subsection{Germanium, $\mathrm{Z}=32$}

In the VALD line list selected here, there are $8 \mathrm{Ge}$ II lines. All of these lines arise from very low lying energy states $(0-0.2 \mathrm{eV})$ with
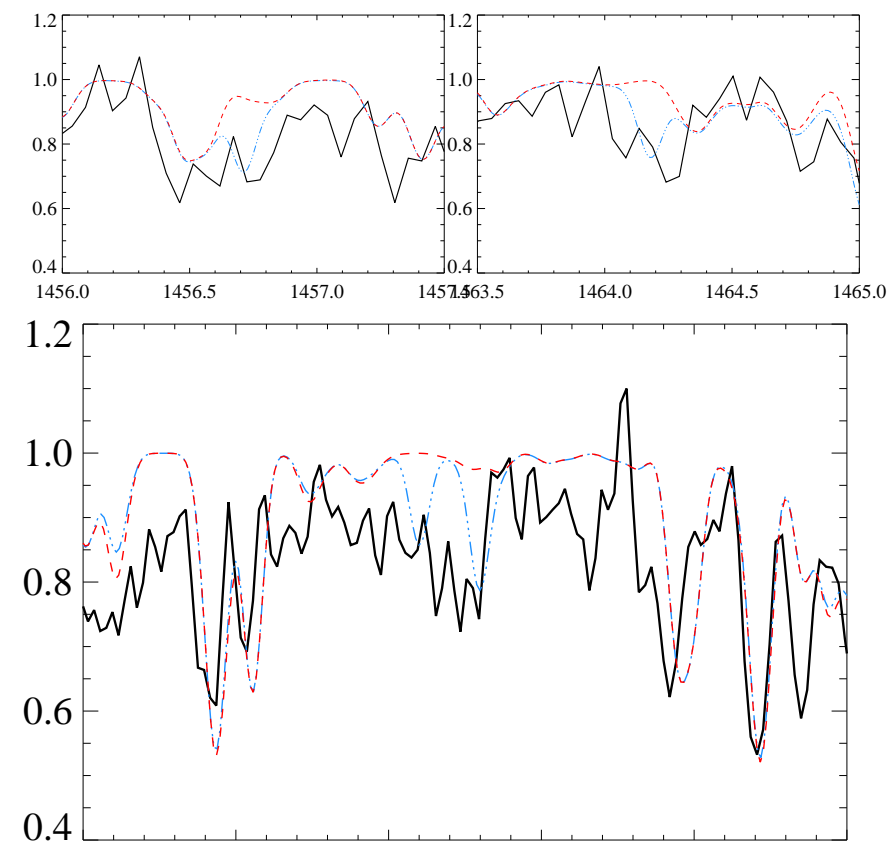

$\begin{array}{llllll}1358.5 & 1359.0 & 1359.5 & 1360.0 & 1360.5 & 1361.0\end{array}$

Figure 21. This figure shows the observation (in black), the calculated model with $\log \left(n_{\mathrm{Zn}} / n_{\mathrm{H}}\right)=-6.85 \pm 0.20$ (in blue), and without zinc (in red). Upper: Zn III lines at 1456 and $1464 \AA$ A. Lower: Zn III lines at $1359 \AA$.

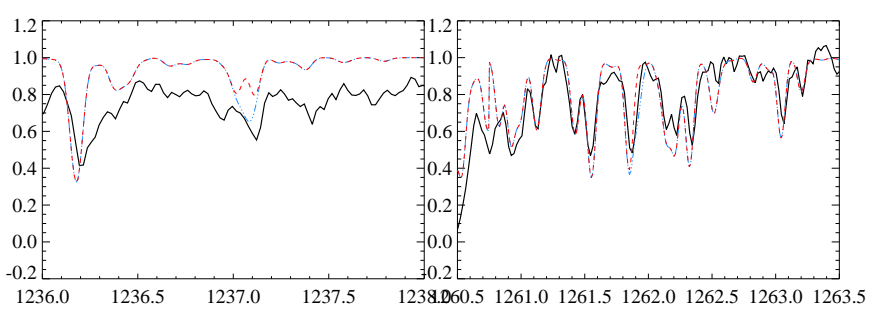

Figure 22. This figure shows the observation (in black), the calculated model with $\log \left(n_{\mathrm{Ge}} / n_{\mathrm{H}}\right)=-8.50$ (in blue), and without germanium (in red). left: A strong Ge II line at $1237 \AA$, right: A part of a Ge II doublet at $1261 \AA$.

$\log g f$ values between 0 to -1 . However, most of these lines are heavily blended which greatly complicates the task of abundance determination. We have used a relatively strong resonance Ge II line at $1237 \AA$ to find $\log \left(n_{\mathrm{Ge}} / n_{\mathrm{H}}\right)=-8.50$. We do no have any other clean lines to confirm this value but a Ge II doublet in 1261-64 region shows that this value can provide an upper limit. Figure 22 shows the model and observation.

\subsection{Platinum, $\mathrm{Z}=78$}

The selection of VALD list used here contains 148 platinum lines. All of them are in the form of Pt III. There is only one Pt II line in the selected list. The dominant state of ionization as estimated by the Saha equation (see Table 3), is consistent with what is observed in the spectrum of $\iota$ Herculis.

There are only a few weak lines throughout the spectrum with a minor concentration at shorter wavelengths. We have used a fairly clean Pt III line around 999 $\log \left(n_{\mathrm{Pt}} / \mathrm{H}\right)=-8.825$. Results are shown in Figure 23 This value 


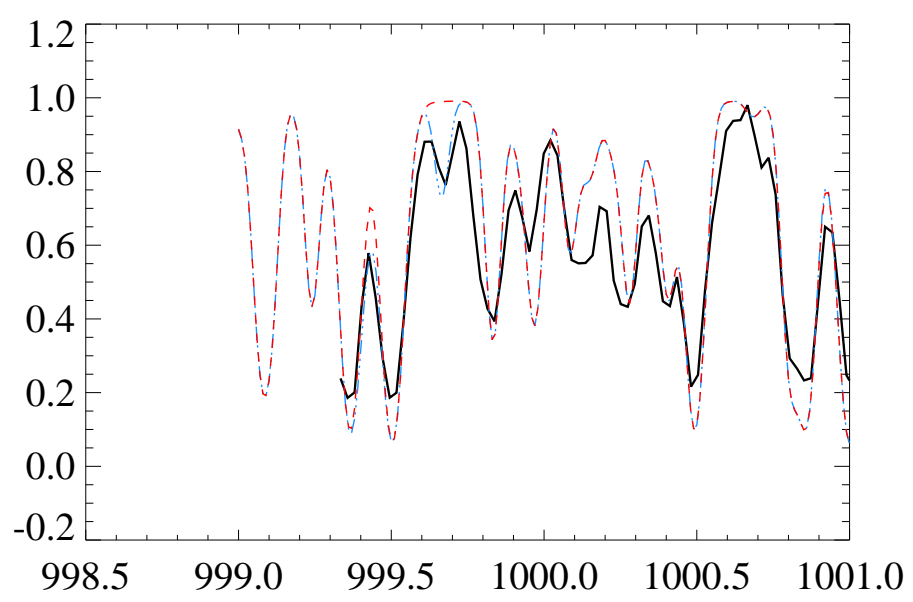

Figure 23. This figure shows the observation (in black), the calculated model with $\log \left(n_{\mathrm{Pt}} / n_{\mathrm{H}}\right)=-8.825$ (in blue), and without platinum (in red). This is the Pt III line at $999 \AA$.

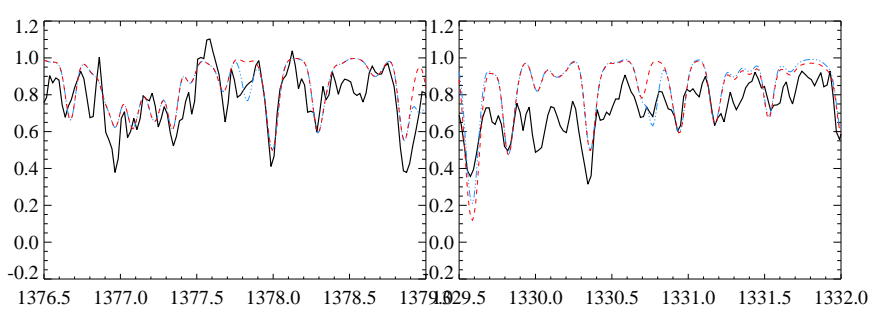

Figure 24. This figure shows the observation (in black), the calculated model with $\log \left(n_{\mathrm{Hg}} / n_{\mathrm{H}}\right)=-8.95 \pm 0.13$ (in blue), and without mercury (in red). Left: $\mathrm{Hg}$ III at $1377 \AA$, Right: $\mathrm{Hg}$ III at $1330 \AA$

was not confirmed with any other line since there are no other clean and unblended lines available throughout our data. Therefore, we present this value as an upper limit for this element for which we can not determine an uncertainty.

\subsection{Mercury, $\mathrm{Z}=\mathbf{8 0}$}

The subset of the VALD database selected here contains 13 mercury lines. In the observed spectrum of $\iota$ Herculis, the majority of mercury lines are observed as $\mathrm{Hg}$ III with a minor contribution from $\mathrm{Hg}$ II. In our wavelength range, apart from two resonance lines, the rest of $\mathrm{Hg}$ II lines arise from higher energy states of around $\sim 7 \mathrm{eV}$. At this temperature, the Saha equation also predicts the same ionization distribution.

There are only very few mercury lines suitable for our purpose and they are mostly blended. We used the $\mathrm{Hg}$ III line at $1377 \AA$ and $1330 \AA$ and we find $\log \left(n_{\mathrm{Hg}} / n_{\mathrm{H}}\right)=-8.95 \pm 0.13$. Figure 24 shows the resulting model and the observation. 


\section{APPENDIX B}

In this appendix, we present the results of the spectrum synthesis. The spectrum in black is the observation and the model spectrum calculated using the final abudances given in the text, is shown in blue. 

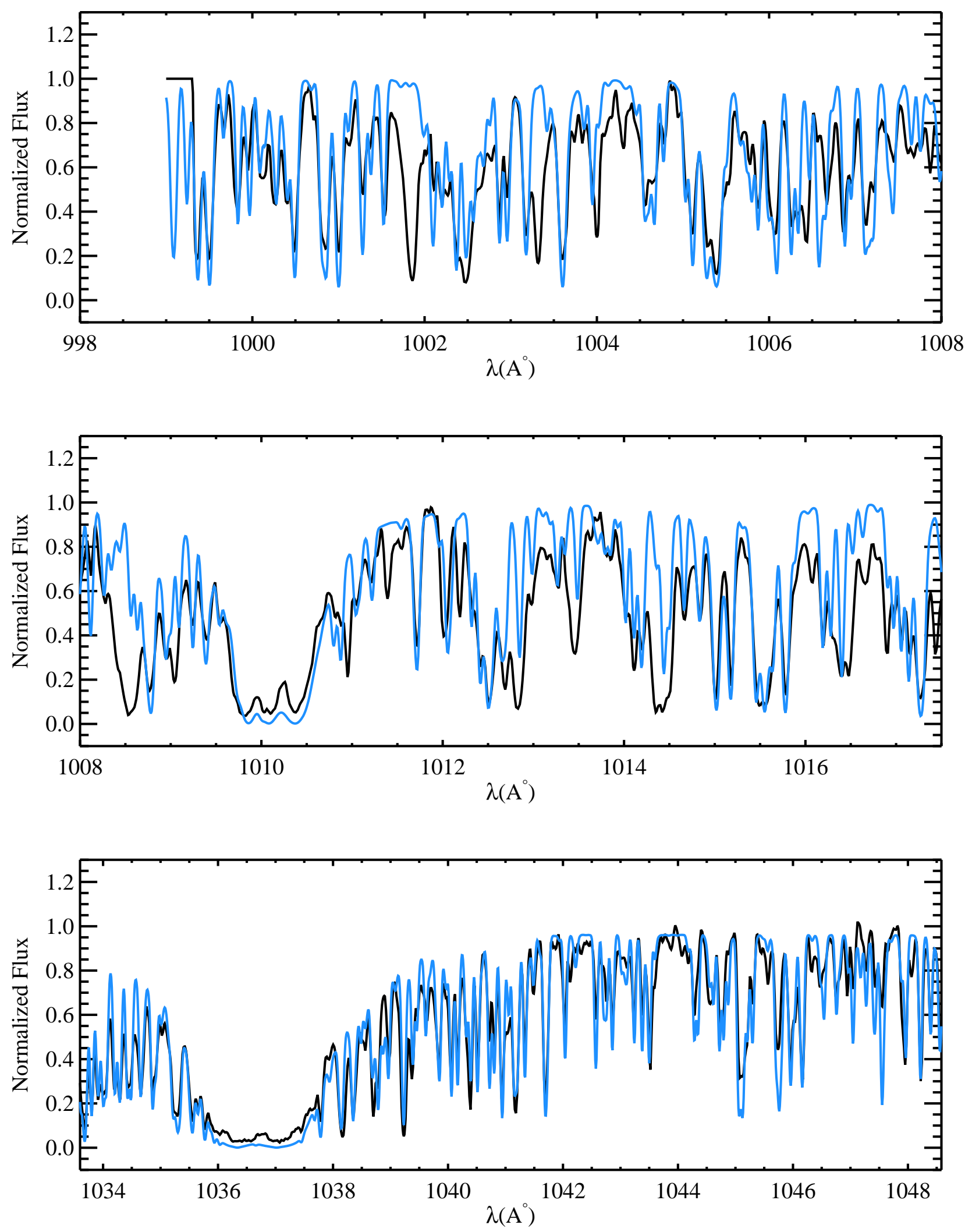

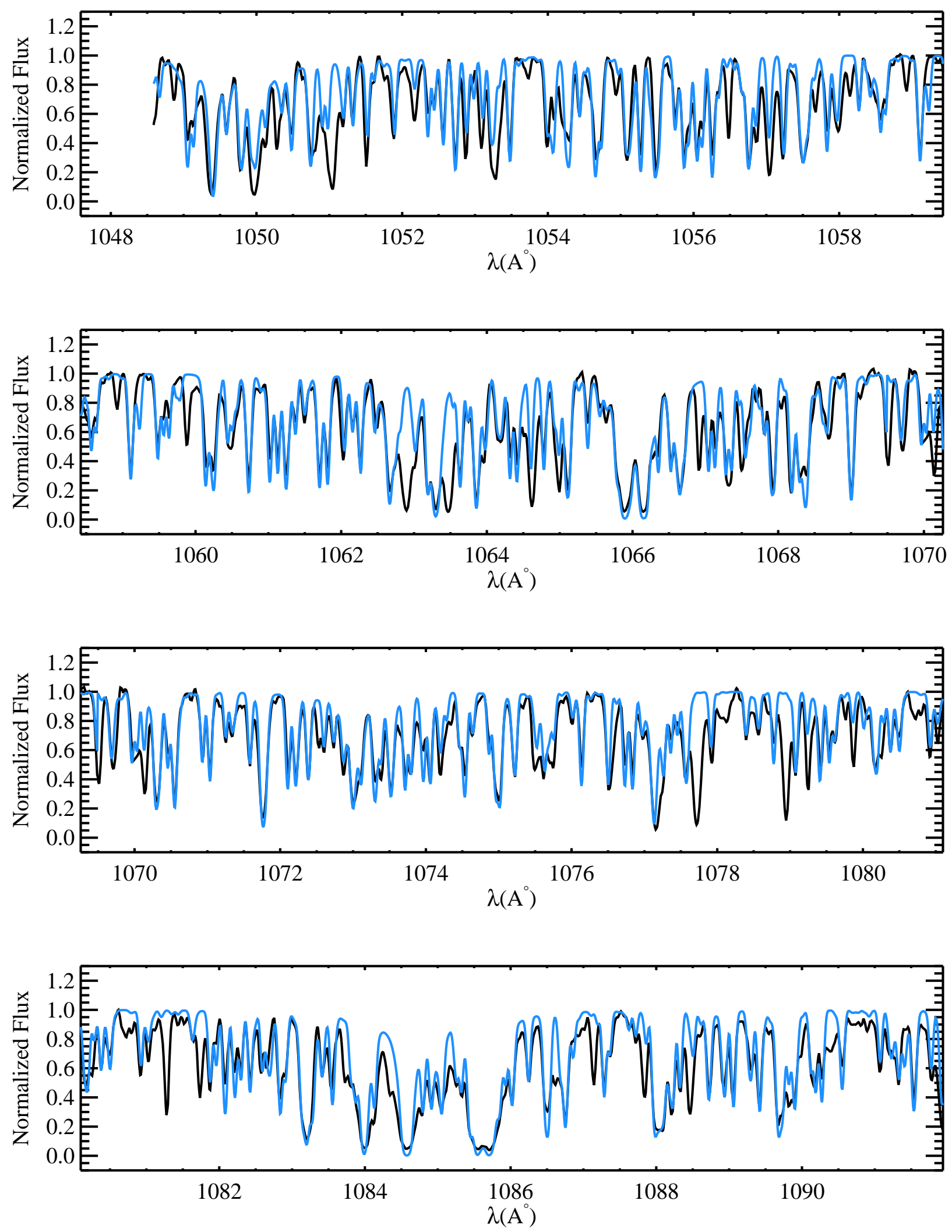

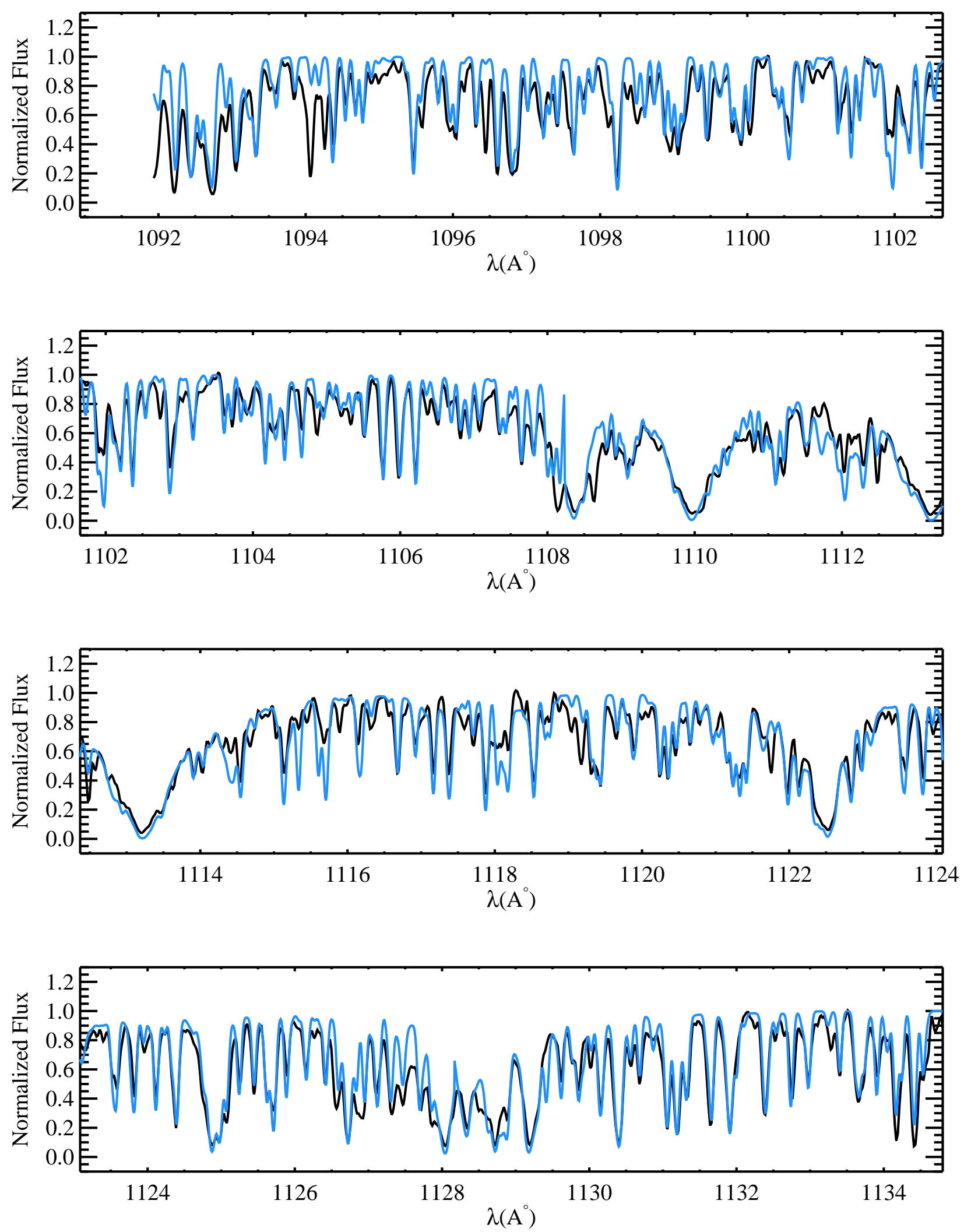

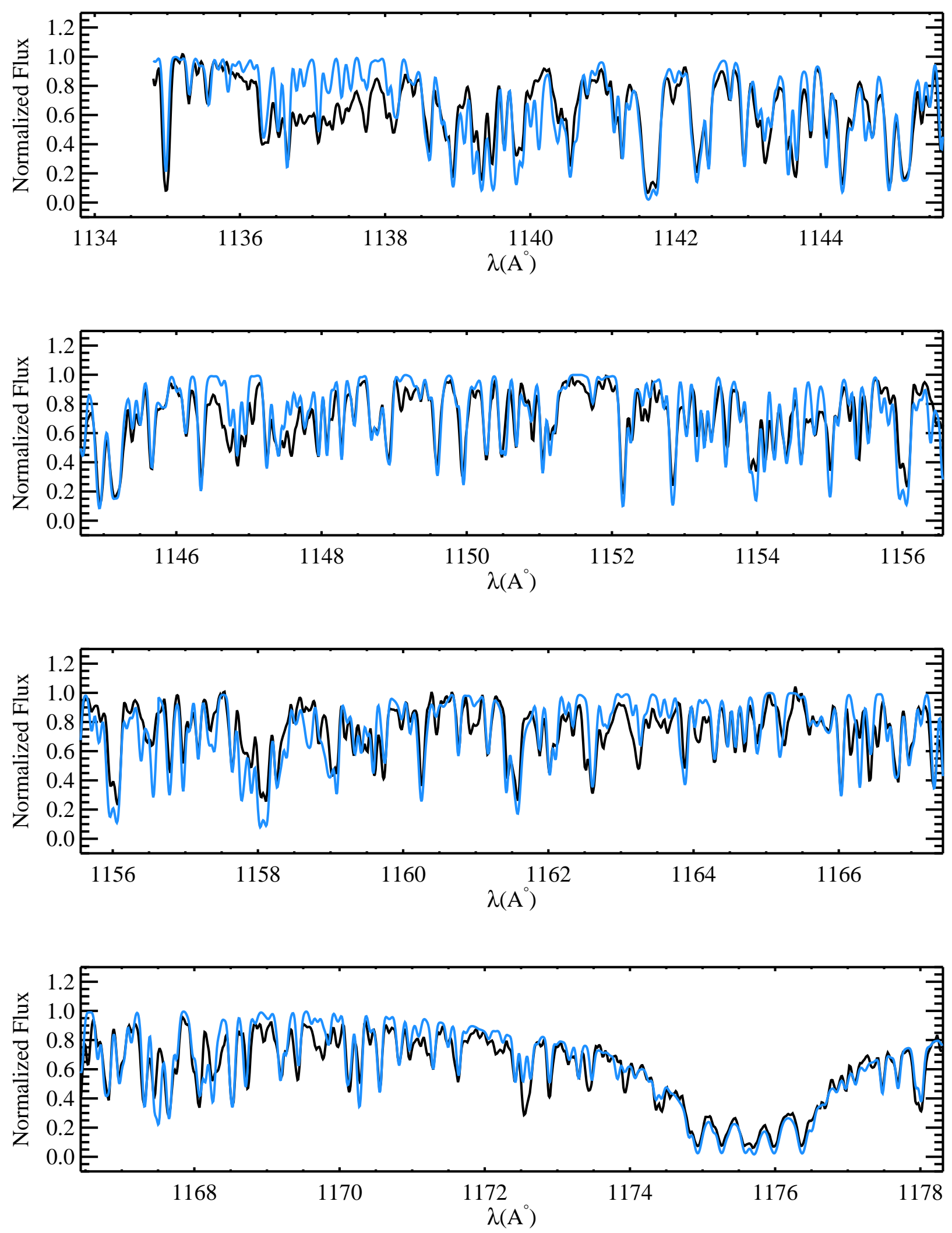

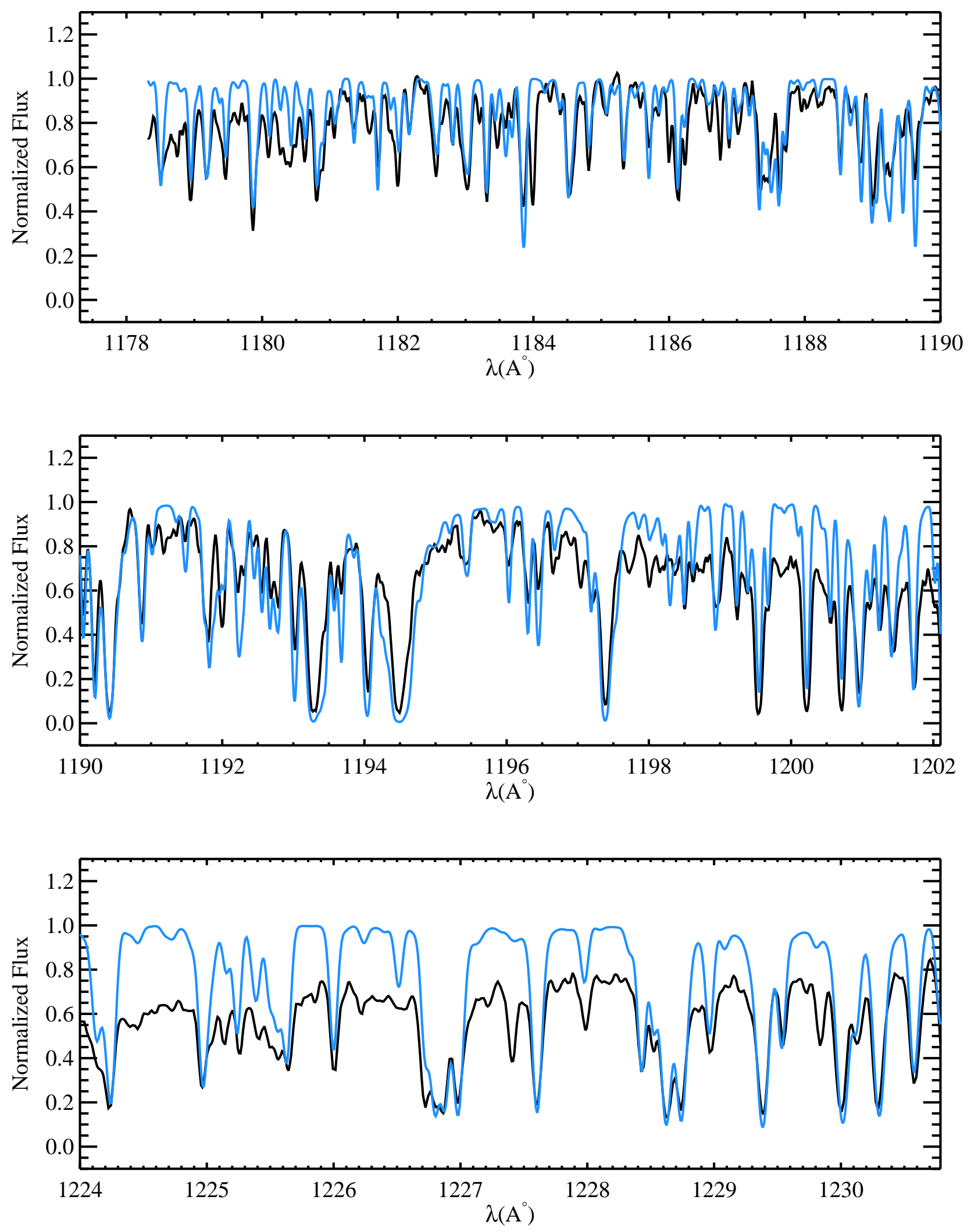

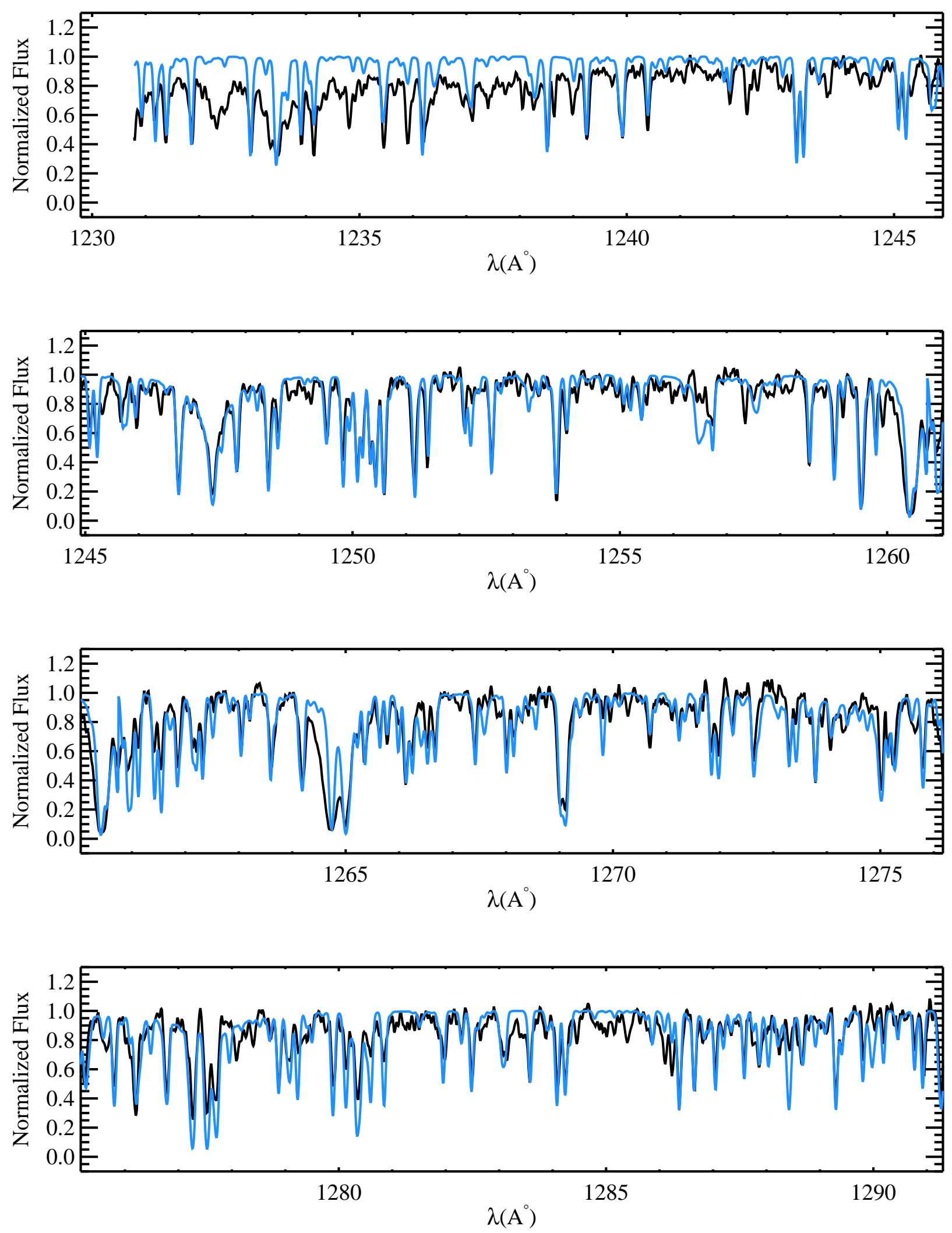

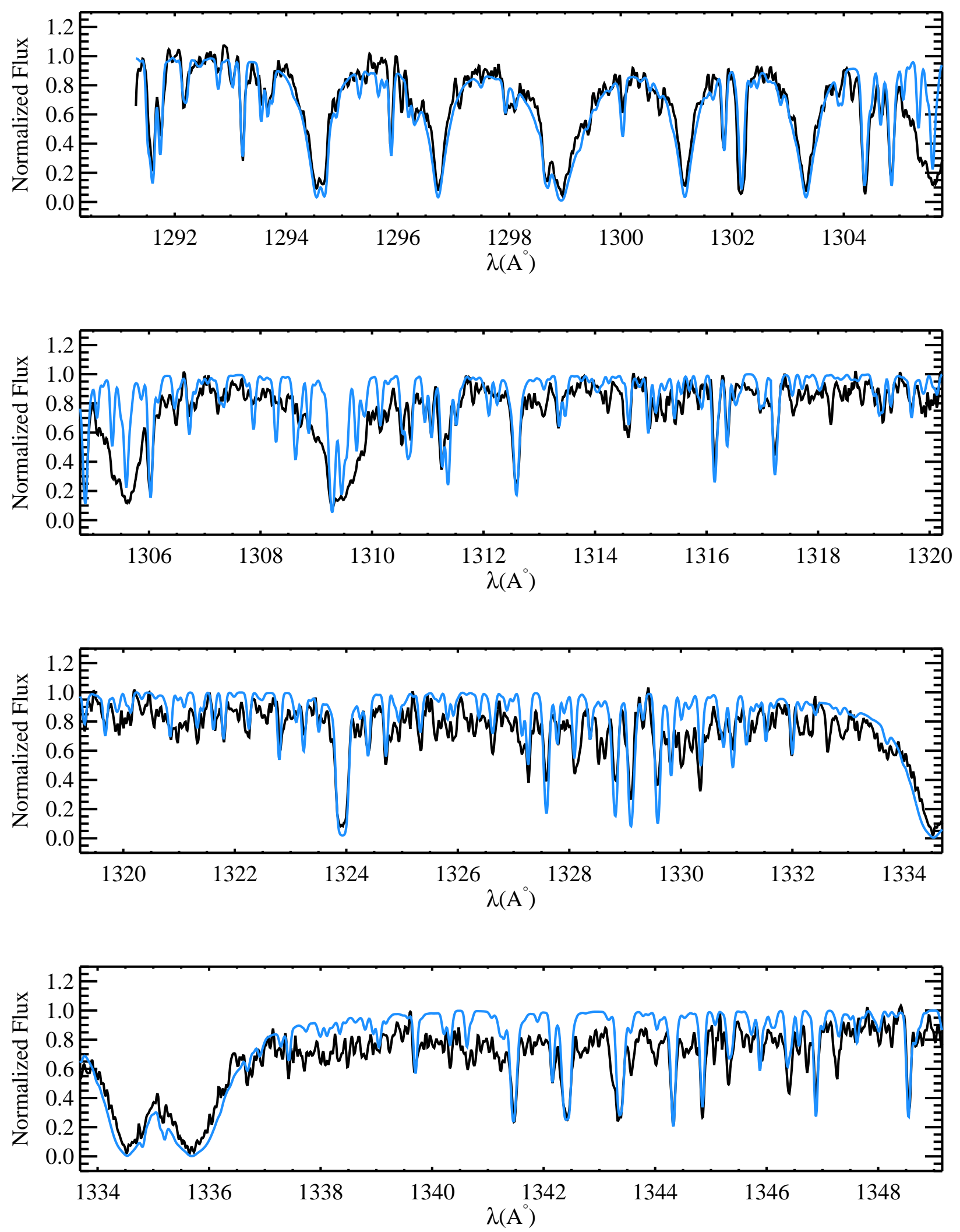

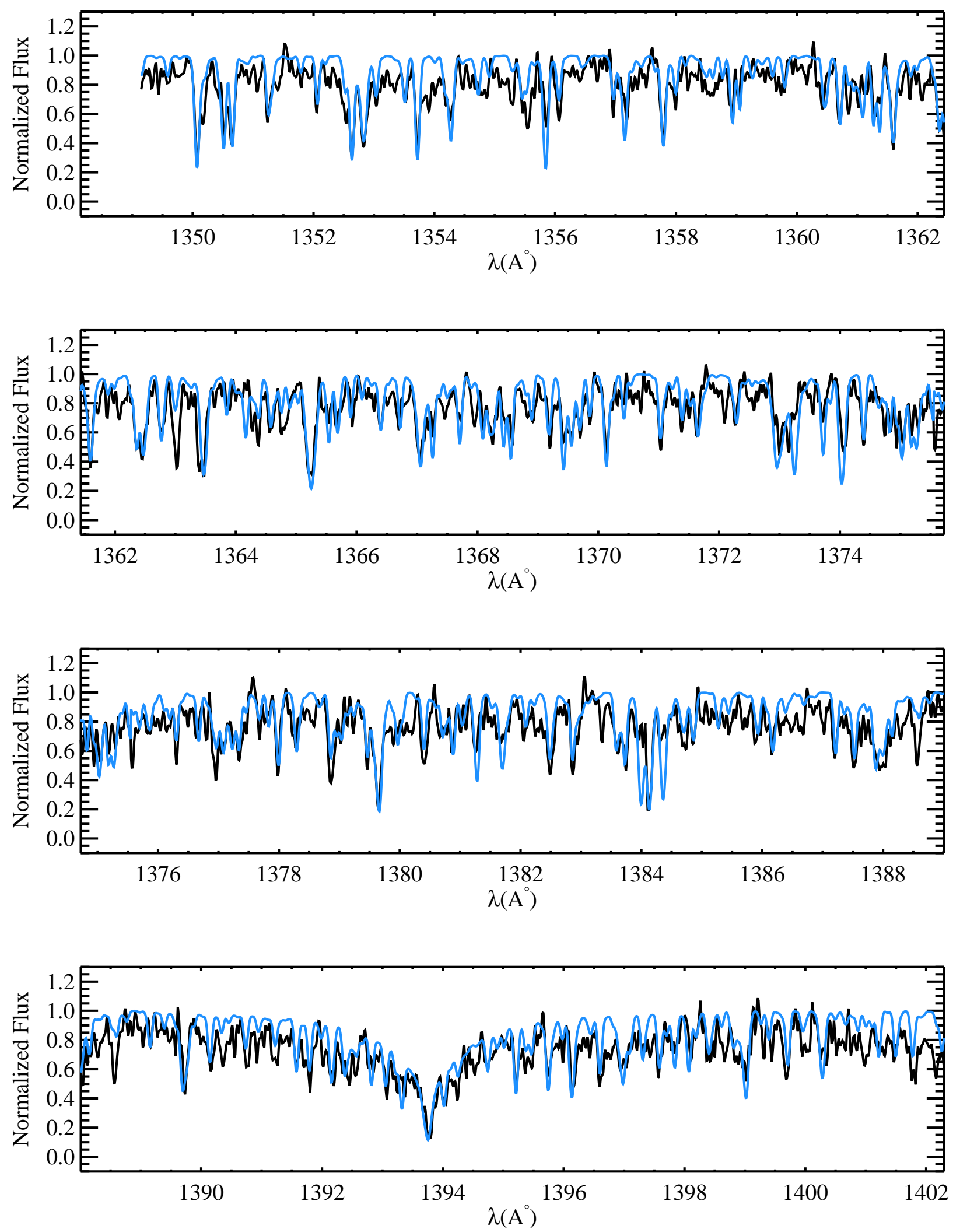

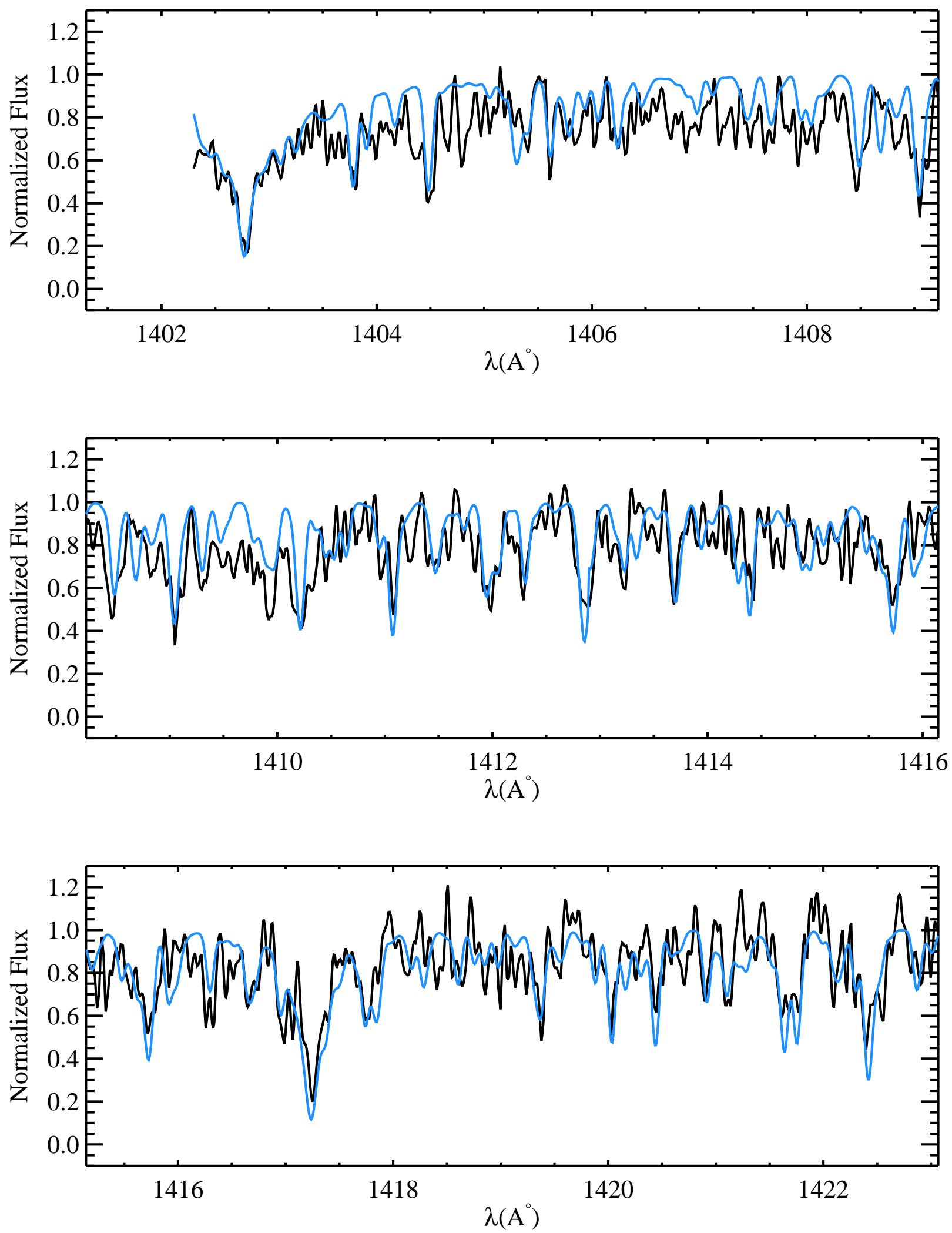

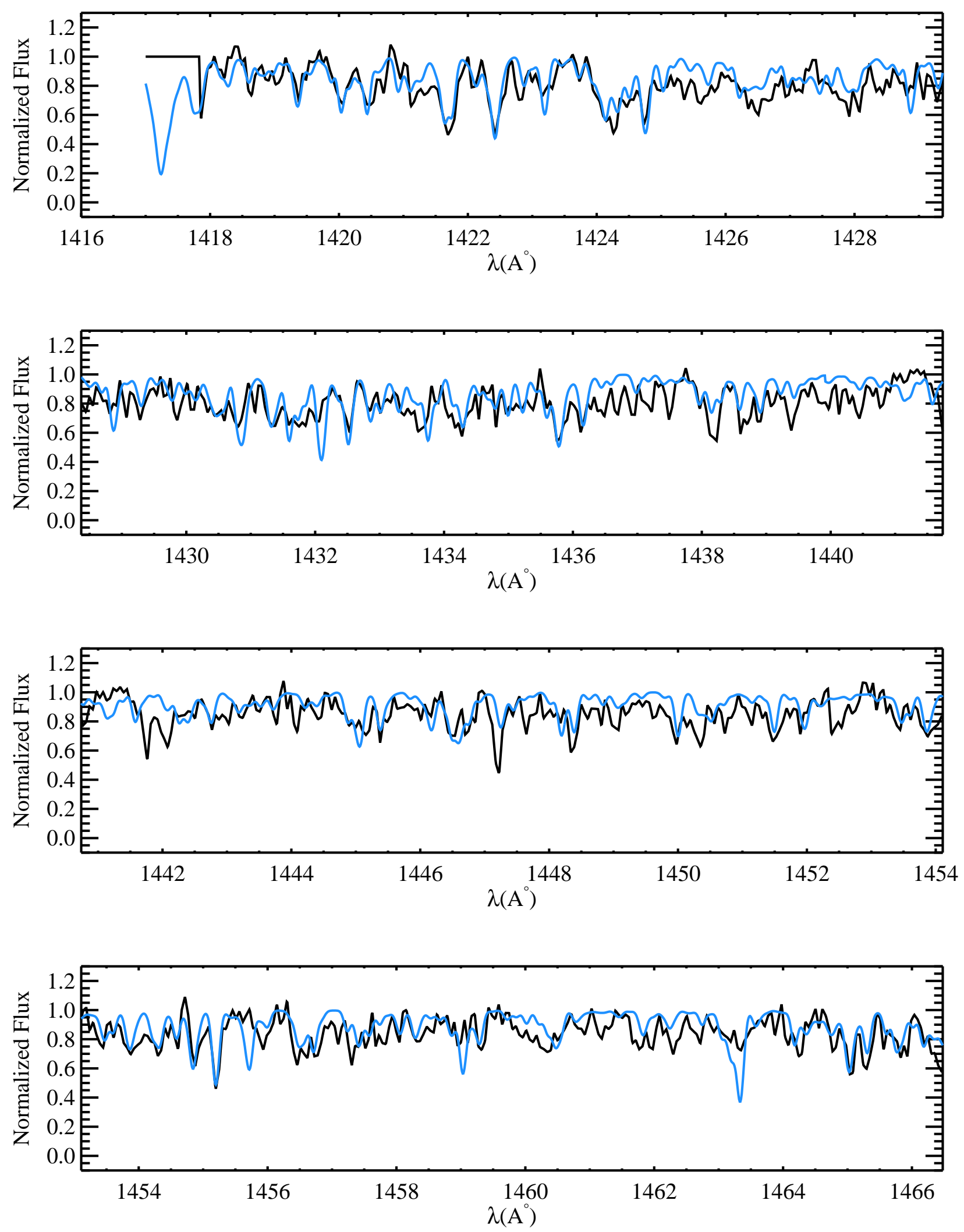


\title{
Ultraviolet Spectral Synthesis of Iota Herculis
}

\author{
S.S. Golriz ${ }^{1}$, J. D. Landstreet ${ }^{1,2}$ \\ ${ }^{1}$ Department of Physics \& Astronomy, The University of Western Ontario, London N6A 3K7, Canada \\ ${ }^{2}$ Armagh Observatory, College Hill, Armagh BT91 6DG, Northern Ireland
}

January 302015

\begin{abstract}
The atmospheric abundances of elements provide essential insights into the formation and evolution history of stars. The visible wavelength window has been used almost exclusively in the past to determine the abundances of chemical elements in B-type stars.

However, some elements do not have useful spectral lines in the visible. A high resolution spectrum of $\iota$ Herculis is available from 999 to $1400 \AA$. In this project, we investigate the abundance determination in the UV. We identify the elements whose abundances can be tested, and search for elements whose abundances can be determined in the UV to add to those in the current literature. We also investigate the completeness of the VALD line list in this region, and the adequacy of LTE modeling in the UV for this star. We have used the LTE spectrum synthesis code ZEEMAN to model the UV spectrum of $\iota$ Herculis for elements with $5 \leqslant Z \leqslant$ 80. Abundances or upper limits are derived for 24 elements.

We find that most of our results are in reasonable agreement with previous results. We estimate a value or an upper limit for the abundance of nine elements in this star that were not detected in the visible.

LTE UV spectral synthesis is found to be a useful tool for abundance determination, even though limitations such as incomplete and uncertain atomic data, uncertain continuum normalization and scattered light, and severe blending can introduce difficulties. The high abundance of two heavy elements may be a sign of radiative levitation.
\end{abstract}

Key words: stars: abundances, ultraviolet: stars, stars: atmospheres, stars: early-type

\section{INTRODUCTION}

' The star $\iota$ Herculis (HD 160762, HR 6588; B3 IV) is a slowlyrotating early B-type star. Such stars are of great interest for ele' mental abundance analysis, since their initial chemical abundances remain fairly intact throughout their short lifetimes, and thus these abundances also constrain the chemistry of the local interstellar medium. Although B-type stars are usually rapid rotators ( $v \sin i \gtrsim$ $100 \mathrm{~km} / \mathrm{s}), \iota$ Herculis is an exception. Its very low rotational velocity makes this star particularly suitable for the measurement of weak and/or blended spectral lines.

Over the past four decades, the optical spectrum of $\iota$ Herculis has been exhaustively studied; the literature now approximately agrees on the values of effective temperature, surface gravity and rotational velocity (see Grigsby et al. 1996).

The literature contains numerous studies regarding the chemical composition of $\iota$ Herculis (see e.g. Peters \& Aller 1970; Kane et al.|1980; Dufton et al. 1981; Lennon 1983; Balona 1984; Peters \& Polidan 1985; Lester et al. 1986; Barnett \& McKeith 1988; Grigsby 1991; Pintado \& Adelman 1993; Grigsby et al. 1996; Nieva \& Przybilla 2012). Table 1 summarizes these abundance determinations.

The measurements listed in Table 1 have mostly been carried out at visible wavelengths except for those of Peters \& Polidan
(1985) who studied the abundances of $\iota$ Herculis in the ultraviolet, using the same spectrum as this project, and reported near solar abundance for all the elements they identified.

Since then, however, the literature has not yet converged into a fully consistent set of abundances, and the number of elements included in the analyses is rather limited. Therefore, a complementary study of this target may be very useful and potentially improve the situation.

In this paper we model a high resolution, high signal-to-noise ratio $(\mathrm{S} / \mathrm{N})$ spectrum of $\iota$ Herculis covering almost the full spectral window from 999 to $1400 \AA$, in an attempt to improve our knowledge of the elemental abundances in this star. In spite of being publicly available data, this ultraviolet spectrum has not previously been used for modern analysis. We will further investigate: (1) the usefulness of full spectrum synthesis of small windows as a tool to measure abundances in crowded spectral regions; (2) the accuracy and completeness of the line-list in the UV for spectrum synthesis in this wavelength region; (3) the accuracy of abundance measurements that can be achieved in the UV in comparison to abundances determined in the optical in the previous literature; (4) abundance values of elements that are accessible in the UV but have not been previously measured in the literature. Finally (5) we try to understand how well LTE synthesis works at this temperature in the UV.

Section 2 describes the observation and reduction of the data 
in more detail. In Section 3.1 we discuss the modeling methods and tools used in this work. Section 4 provides an introduction to the comprehensive element-by-element discussion of our results (found in the on-line Appendix) as well as a brief discussion of the "missing lines". Finally, in Section 5, we compare our results to the literature and summarize our new results.

\section{OBSERVATION AND DATA REDUCTION}

The results presented in this work are based on the high-spectralresolution ultraviolet observations obtained from the Copernicus Spectral Atlas of $\iota$ Herculis (see Upson \& Rogerson 1980, for details). The Copernicus satellite was the third Orbiting Astronomical Observatory (OAO-3), launched in August 1972; it operated until 1982.

The spectra used in this work contain two orders, covering wavelengths from 999.3-1422.2 (U1, second order) and 1417.9$1467.7 \AA$ (U1, first order). The data have reasonably high spectral resolution of $\lambda / \Delta \lambda \sim 14,000$ in first order, and $\lambda / \Delta \lambda \sim$ 24,000 in second order, and the co-added data reach $\mathrm{S} / \mathrm{N}>100$ Klinglesmith et al. 1996).

The data acquired from the Copernicus spectrometer have been reduced with considerable care, including careful wavelength calibration, corrections for effects due to changes in spectrometer temperature, terrestrial and spacecraft Doppler shifts, background corrections for cosmic rays and charged particles, correction for (estimated) scattered light, and for stellar image drift on the spectrometer slit (see Upson \& Rogerson 1980, for more details).

The total uncertainty of the relative flux presented in the atlas is of the order of 2 or $3 \%$ of the local continuum flux for wavelengths below $1200 \AA$ and rises gradually at longer wavelengths to about $15 \%$ at $1400 \AA$ in the second order spectrum; it is about $10 \%$ throughout the first order spectrum Upson \& Rogerson 1980). Most of this uncertainty is due to statistical fluctuations in the photon counts.

A normalized version of the spectrum is provided in the Copernicus atlas, using a smooth envelope defined by maxima in the Fourier-smoothed spectra. However, we have found that this normalization is not a very accurate representation of the real stellar continuum.

We originally used the normalized atlas spectrum in our modeling routine, but we found a clear mismatch between models and the observations in the sense that the computed high points in the spectrum often lie above the atlas continuum (see 3.1 for details). We resolved this by re-normalizing the observed spectrum in three steps; (1) manually specifying continuum points over intervals of $\sim 20 \AA$, (2) fitting the continuum with the exponential of a cubic spline, and (3) dividing the spectrum by our smooth fit. The re-normalization process was iterated until the least mismatch between the observation and model was achieved (see section 3.1 . However, this process still leaves some uncertainty in the correct location of the continuum, which will be discussed in section 3.3

\section{METHODS}

\subsection{Abundance Determination Method}

To carry out an analysis of a spectrum as crowded as that of $\iota$ Her, in which essentially every spectral feature is blended at least to a minor extent with other features, usually mixing individual spectral lines of more than one element, it is essential to use the methods of spectrum synthesis. A model of the spectrum is computed assuming various basic parameters of the star (effective temperature, etc) and an initial abundance table. The spectrum is computed using a line list that one hopes contains all the significant spectral lines of all elements, including the best atomic data (excitation level, oscillator strength $\log g f$, damping constants) available for each line. Assuming that the basic parameters of the star have already been established, the abundance table is varied until the best computed fit to the observed spectrum is obtained. This may be done by varying a single element at a time, iterating through the elements that contribute to a spectral window, or by varying several elements at once. To the extent that the underlying atmospheric model is appropriate, and the atomic data are accurate, this procedure allows one to establish the abundances of a number of elements even in the presence of severe blending in almost every feature.

In this work, we use the FORTRAN spectral synthesis program ZEEMAN.F (Landstreet 1988; Landstreet et al. 1989; Wade et al.2001). This program was originally designed for optical spectropolarimetry of magnetic stellar atmospheres, but works well (if a little slowly) for non-magnetic stellar spectra as well. (Note that the literature does not contain any conclusive evidence for the presence of magnetic fields in the atmosphere of Iota Herculis. Recent attempts to search for weak or complex magnetic fields in this star have not detected any non-zero Zeeman signatures (Wade et al. 2014).)

To use a program like ZEEMAN that was originally designed for use on spectra obtained in visible light, the main concern is whether all necessary continuous opacity sources are included. The continuum opacity subroutine used by ZEEMAN includes the essential $\mathrm{H}$ and $\mathrm{He}$ bound-free and free-free ultraviolet opacities of $\mathrm{H}^{-}$, $\mathrm{H}$, and $\mathrm{H}^{+}$, and of $\mathrm{He}^{-}, \mathrm{He}, \mathrm{He}^{+}$and $\mathrm{He}^{+2}$. Continuous opacity due to electron scattering and Rayleigh scattering from neutral $\mathrm{H}$ (longward of Lyman $\alpha$ ) are treated as pure absorption. ZEEMAN also includes a small number of ground state and low-lying boundfree transitions of common neutral elements, but none of ions.

ZEEMAN uses stellar atmospheric models produced with the code of Kurucz (1970) with solar composition, precomputed for us by Piskunov \& Kupka (2001), and a set of atomic line data from the Vienna Atomic Line Database(VALD; Piskunov et al. (1995); Ryabchikova et al. (1997); Kupka et al. (1999, 2000)). As input parameters, the program requires the effective temperature $\left(\mathrm{T}_{e}\right)$, gravitational acceleration $(\log g)$, estimated rotational velocity $(v \sin i)$, microturbulence parameter $(\xi)$, and initial individualized abundances, in order to compute the emergent atmospheric spectrum. For our synthesis we used $\mathrm{T}_{e}=17500 \mathrm{~K}, \log g=3.8, \xi=1 \mathrm{~km} / \mathrm{s}$, and $v \sin i=6.0 \mathrm{~km} / \mathrm{s}$ (see Table 5; Nieva \& Przybilla 2012). These parameters were not varied. ZEEMAN is able to synthesise a spectral window of some tens of $\AA$ at once, utilizing a line list of up to about 2000 lines. ZEEMAN can then adjust the abundance of any element of choice through an iterative process until a least squares best fit with (automatically selected) spectral features in the window under study is achieved. This process of course works best for unblended lines for which the abundance can be adjusted without any contamination from other elements.

ZEEMAN was designed for LTE conditions; this is marginally appropriate for $\iota$ Herculis, whose effective temperature is near the boundary at which non-LTE effects start to become important. However, Przybilla et al. (2011) have shown that for the main sequence stars with effective temperatures $(T \lesssim 22000 \mathrm{~K})$ and carefully selected spectral lines, pure LTE modeling can yield meaningful results. They also show that despite the conceptual superiority 
of NLTE modeling, inadequate model atoms can potentially result in larger systematic errors than LTE modeling.

In this work, we use LTE modeling, even though an ideal global match between synthetic and observed spectra can only be achieved when the NLTE effects on the lines are fully taken into consideration. We used a spectral line list retrieved from VALD covering wavelengths 900 to $1500 \AA$, selected for significant depth at the effective temperature and gravity of $\iota$ Her. The elements included in the resulting line list in this spectral range, assuming solar abundance, are all listed in Table 2

We extracted line data from the VALD database using the Extract Stellar option. The criteria we used to select our linelist were the following: starting wavelength: $900 \AA$, ending wavelength: $1500 \AA$, detection threshold: 0.01 of the continuum with $v \sin i=0$, microturbulence: $1 \mathrm{~km} / \mathrm{s}, \mathrm{T}_{\text {eff }}: 17500 \mathrm{~K}$, and $\log g$ : 4 (in cgs units). We requested long extraction format in October, 2014.

As discussed in general above, one of the main challenges in using a crowded spectral region for abundance analysis is to identify spectral features that respond primarily to the abundance of a single element. ZEEMAN has a mode for determination of abundance of elements one at time, in which the program first tries to identify features that respond primarily to the abundance of the element being fit, and then iteratively modifies the abundance until a best least squares fit is achieved to the features, or parts of features, identified as sensitive primarily to that element. The identification of such features is carried out by computing two trial synthetic spectra of the observational window being fit, one with an assumed abundance of the element being fit that is slightly enhanced compared to the final value expected (or guessed), and a second computed with the element entirely absent. ZEEMAN compares these two spectra and then selects individual wavelengths in the model spectrum to use for fitting based on simple criteria (which can be adjusted by the user by modifying a couple of lines of code). Two examples of criteria used would be to require (1) that any blending feature (i.e. still present in the spectrum with the fitted element removed) be no deeper than 0.95 of the continuum, and that the depth of the feature with the desired element present be deeper than 0.7 of the continuum, or (2) that the line be deeper than 0.7 of the continuum, and more than 4 times deeper than blending features.

After selecting a list of wavelength grid points in the computed spectrum based on such criteria, ZEEMAN iterates the abundance of the desired element to find a least squares best fit to the selected wavelengths, using a downhill simplex method. At the end of the process, the value of the abundance which produces the best fit is reported, along with a plot file of the current best fit spectrum for the window, the initial spectrum without the element being fit, and a map of wavelengths used in the fit. Graphical visual examination of these spectra enables the user to decide if the program has actually found useful spectral features or not, to decide if the fit found is based on strong enough evidence to consider that the reported value is useful, and to see whether the best fit is consistent over the various features available in the window, whether explicitly fitted or not. Many such fits to small windows, comparing the observed spectrum to both the model spectrum with a best fitting abundance, and also to a spectrum with the fitted element absent (e.g. Figure 1), will be used in this paper to illustrate our results. The examination of such comparison spectra is an extremely useful technique for studying synthetic spectral models of crowded spectral regions.

Using the techniques outlined above, we find that several elements exhibit a few apparently clean and unblended lines. After identifying those lines, we used them to adjust the abundances of those elements (see $\$ 4$ ). Table 2, which presents the abundance values (ion by ion) that are found to best fit the observed spectrum, lists the lines that we found to be most useful.

For some elements of interest, reasonably strong lines are present in our spectrum, but most are not really free of blends. The analysis of blended lines is slightly more challenging since they can have more than one element contributing to the overall shape of the line profile. In these cases, a few more steps are required. We first iterated over the abundance of each element, assuming solar abundance for the rest in order to identify which wavelength window could best be used to adjust the abundance of that particular element. This first approximate model gives us a first estimate of the abundance even though it is not the final result. Once the first iterations are carried out, we are left with an initial set of model parameters. Having selected the most useful window(s) for each element, we then iterated the abundances of both the element of interest and also the blending element(s) until we obtained the best possible global fit to the observation.

The computed model spectrum, using the final set of abundances, results in a reasonably good fit to the observed spectrum (see Appendix B), although it is quite clear that there are lines in the observed spectrum that are missing from the VALD list. One significant source of such lines is due to absorption by the Lyman bands of interstellar $\mathrm{H}_{2}$.

\subsection{Line Broadening}

Because we are trying to fit individual spectral features as accurately as possible in spectra of fairly high resolution, we must take into account both local line broadening (natural broadening, van der Waals and Stark broadening, which are included in the computation of the local line profile opacity coefficients as a function of depth in the atmosphere), and broadening due to various velocity fields, including micro- and macroturbulence, pulsation, and rotation.

The microturbulent velocity $\xi$ is the non-thermal component of the local gas velocity in the spectral line formation region of the stellar atmosphere (Cowley 1996). The validity of this concept has been the subject of debate for decades. For instance, it has been suggested that the use of non-LTE in spectral analysis should eliminate the need for microturbulence. However, it is found by Nieva \& Simón-Díaz (2011) that consistent fitting of many spectral lines in early B stars with an advanced non-LTE code still requires the introduction of microturbulence. It is suggested that the origin of the photospheric microturbulence in hot stars is the sub-surface convection. Convective regions in the envelope of a hot massive star are generated by the opacity peaks of ionized iron and (nearer the surface) of the He II to He III ionization zone (see Cantiello et al. 2009, for more details). (There may also be very weak convection at the $\mathrm{He}$ I to He II ionization zone, which is within the visible atmosphere.)

Nieva \& Przybilla (2012) have carefully carried out an extensive non-LTE analysis of high-resolution optical spectra for a sample of early B-type stars including $\iota$ Herculis. Through an iterative process, they have constrained the stellar parameters including the microturbulence. They find a microturbulence of $\xi \sim 1 \mathrm{~km} / \mathrm{sfor}$ $\iota$ Herculis. We have included their result in our calculations.

In contrast to microturbulence, macroturbulence is the photospheric velocity field with scales much longer than the mean free path of the photons. This broadening component changes the overall shape of spectral lines from roughly Gaussian (when thermal and microturbulent broadening dominate) or U-shaped (when rotational velocity broadening dominates) to a more triangular form. It 
has been proposed that macroturbulent broadening in hot stars may be the result of many low-amplitude pulsation modes (Aerts et al. 2009). Since $\iota$ Her is known to be a slowly pulsating B star (Chapellier et al.2000), we might expect to find some indication of macroturbulence in the line profiles. However, Nieva \& Przybilla (2012) showed that in case of $\iota$ Herculis, macroturbulence is negligible, and the spectral profiles can be reproduced with only rotational broadening and microturbulence. The small (a few $\mathrm{km} / \mathrm{s}$ ) and quite slow excursions in radial velocity due to the pulsations do not seem to have led to obvious radial velocity inhomogeneity in the available Copernicus spectra, perhaps because each short scan was corrected for radial velocity individually.

The projected rotational velocity of the star around its axis, $v \sin i$, is difficult to measure accurately because it is not much larger than typical values of other velocity fields. It is necessary to take into account the impact of microturbulence as well as macroturbulence when measuring the $v \sin i$ values (Simón-Díaz \& Herrero 2014). The $v \sin i$ value of $6 \mathrm{~km} \mathrm{~s}^{-1}$, determined by Nieva \& Przybilla (2012), is used here..

In fact, in the Copernicus spectrum, with a resolving power of at most only about 24000 , which is not sufficient to fully resolve the rotational velocity, instrumental broadening is the dominant line broadening mechanism. We find that combining the various broadening mechanisms above with the instrumental profile (assumed Gaussian in ZEEMAN) yields a satisfactory fit to observed quasiisolated lines in the spectrum.

\subsection{Sources of Uncertainty}

The stellar parameters used here (e.g. $T_{\text {eff }}$ and $\log g$ ), of course, are not exact but have relatively small uncertainties. The uncertainties associated with $T_{\text {eff }}$ and $\log g$ are about $\pm 200 \mathrm{~K}$ and \pm 0.05 (cgs), respectively (see Table 5; Nieva \& Przybilla 2012). We find that an increase of 0.05 in surface gravity would result in an increase of typically about 0.02 in the abundance, and the rise in temperature by $200 \mathrm{~K}$ will cause the abundance to increase by 0.035 . Therefore, the uncertainty arising from uncertain stellar parameters is roughly \pm 0.04 .

Another, probably more important, source of error is the renormalization of the spectrum (2). In order to evaluate this uncertainty, we have used multiple spectral lines of one atom and attempted to determine abundance for each of them separately (see e.g. nickel $\S$ Appendix A).

A similar source of error is the correction for background and scattered light, which sets the zero level of the spectrum. In some parts of the spectrum, the strongest computed lines are deeper than the observed lines (for example in the cores of the $\mathrm{C}$ II resonance doublet of UV multiplet (1) at $1335 \AA$ ), suggesting that the background correction should be a little larger than the one used by Upson \& Rogerson (1980). We have not tried to correct for this effect. The uncertainty actually introduced by this effect is small because in lines deep enough for this to be a significant source of error, we frequently have fitted the (sometimes rather broad) damping wings rather than the core depth.

In some cases, the best-fitting models of several individual lines or multiplets will not result in the same abundance value for that element. This may be produced by the effect of incorrect continuum normalization, lines missing from our VALD line list, inaccurate atomic data, and/or non-LTE effects of under- or overionization of minority ionization stages (Lanz \& Hubeny 2007).

The effects of missing blending lines can sometimes be identified by careful comparison of the observed and calculated line pro- files. It is not possible to generalize about the uncertainty resulting from this problem, but in a few cases we can include an estimate of the effect in our uncertainty estimates.

The atomic data uncertainties for individual lines can be estimated in some cases from the literature, especially for resonance lines for which very accurate theoretical and/or experimental oscillator strengths are sometimes available (for example, according to the NIST database (Kramida et al. 2014) the accuracy of the oscillator strengths of the C II resonance lines of multiplets (1) (at $1335 \AA$ ) and (2) (at $1036 \AA$ ) is "A", with uncertainties of about $\pm 3 \%$ or $0.01 \mathrm{dex}$ ).

For most of the line data in VALD, very accurate oscillator strengths are not available (typical uncertainties for logarithmic oscillator strengths of a light ion such as Si II are of the order of \pm 0.1 dex, while those of Fe II lines are of the order of \pm 0.2 dex, for example). These uncertainties are similar to those for the optical lines often used for abundance analysis (e.g. Ryabchikova et al. 1994), and probably set an important lower limit to uncertainty. Except when we use lines of very well determined oscillator strengths, we will assume that atomic data introduce uncertainties of this order.

Non-LTE effects can alter the relative populations of various levels of a single ionization stage, and also the relative numbers of different ionization stages. We do not have any very useful method for identifying such effects within a single ionization stage except by looking for large discrepancies between different "reliable" lines of that ionization stage, and comments in the literature about individual lines and multiplets. In contrast, we expect the non-LTE effects on relative numbers of different ionization stages to affect primarily the ionization stages with relatively small populations. In order to identify the sensitive stages, we have computed (in LTE) the relative populations of various ions of interest for temperature - electron density values appropriate to about continuum optical depth (evaluated at $5000 \AA$ ) $\tau_{\text {cont }} \sim 0.2$ and $\sim 0.001$. The results are given in Table 4 . We believe that abundances derived for ionization stages that in LTE account for at least roughly $10 \%$ of the total population of an element are not very sensitive to under- or overionization effects, but that less populated ionization stages may be. Thus we may be able to identify a few cases of non-LTE ionization effects by finding systematic discrepancies between abundances derived from dominant ionization stages and from weakly populated stages.

In a general way, we expect that the effects discussed above will lead to uncertainties in the derived (logarithmic) abundances of the order of \pm 0.3 dex or perhaps somewhat more (or less) in some cases. We are sometimes able to estimate the abundance uncertainties of individual atoms by looking at the dispersion of results obtained from different multiplets, and the scatter is indeed often of this amplitude.

\section{SUMMARY OF INDIVIDUAL ELEMENTS}

In this section, we present sample results for a few element identified in the spectrum of $\iota$ Her. We have used several spectral lines for abundance determination, in order to reduce the bias due to the kinds of uncertainties discussed above. The final abundances determined for individual ionization stages of these elements are listed in Table 2 We have correspondingly omitted from our modeling a few $\AA$ on either side of Lyman $\alpha(1215.67 \AA)$ and Lyman $\beta$ (1025.72 $\AA$ ). In fact the wings of these two lines extend to approximately \pm 20 and $\pm 15 \AA$ from line center respectively. ZEE- 
MAN has not been enabled to compute hydrogen lines correctly, although there would be no difficulty in principle with this. The modification of the code and the proper analysis of these lines will be the focus of future work. We have modeled strong lines in the farther wings of these two lines in spite of the omission of Lyman line wing opacity, because the opacity of strong lines so completely overwhelms the opacity of the Lyman line wings (important mainly in the deeper atmosphere layers in any case) that the line strengths are largely independent of the neglect of the Lyman line wings. In fact the syntheses of the strong metallic lines in the Lyman line wings are surprisingly successful.

It is worthwhile to mention that iron provides strong line opacity over the entire wavelength range studied here. Probably most of the lines of other elements are at least slightly blended with lines of iron. Therefore, iron was the first element for which we determined the abundance (see section $\S 1.17$ in Appendix A). However, in what follows we only provide a few examples of these results. Figure 1 shows a sample of our results for three elements carbon, boron, and germanium. In case of carbon, we have a relatively clean and unblended set of multiplets which allows a clear determination of the abundance value. In contrast, in the case of germanium or boron, the lines are not unblended and thus are more suitable for determination of an upper limit for the abundance. We refer the reader to Appendix A for a detailed discussion of each element studied. Table 3 contains a list of the spectral lines that we found particularly useful for this project, with the adopted $g f$ values and lower ionisation potentials. Table 2 provides the derived abundance and upper limits for all the elements studied here.

Notice in Figure 1 how our technique of visually (and within ZEEMAN, numerically) comparing a spectral region with and without the element of interest allows $u$ to easily assess how useful a region is for abundance determination. This method relies simply on the availablity of a relatively complete line list, such as those provided by VALD. In fact, such comparison allow us to test line lists and in some cases to identify lines in the list with seriously incorrect atomic data (see the example of Si I in Sirius A (Landstreet 2011)).

\subsection{The Missing Lines}

We have done the spectrum synthesis from which we have derived abundances using the atomic data provided in the VALD database. However, the spectrum of $\iota$ Her still contains some fairly strong lines that are partially or entirely missing from the calculated model (see e.g. $1001.85 \AA, 1003.33 \AA, 1051.05 \AA, 1062.9 \AA, 1077.72 \AA$, $1078.95 \AA$, etc. in Appendix B).

One source of lines missing from our synthesis is lines due to interstellar absorption by the Lyman bands of the $\mathrm{H}_{2}$ molecule. The transitions that affect our data are the resonance lines of electronic transitions between the ground electronic-vibrational state (the normal state for interstellar $\mathrm{H}_{2}$ below about $30 \mathrm{~K}$ ) and the low vibrational states of the first electronic state. The relevant wavelengths are provided for example in Table 4 of Swamy \& Tarafdar (1972). These absorption lines are all present in $\iota$ Her, in some cases clearly coinciding with strong observed lines for which our synthesis has no match whatever $(1001.8 \AA, 1062.9 \AA, 1077.1 \AA$, in other cases also coinciding with strong absorption lines, but with ones for which stellar lines in our synthesis fill a part of the profile (1012.8 $\AA, 1092.2 \AA)$. It is quite clear that $\mathrm{H}_{2}$ Lyman band interstellar absorption contributes a number of significant lines to the observed spectrum of $\iota$ Her below 1108 Å.

We also investigated the possibility that a few of the missing
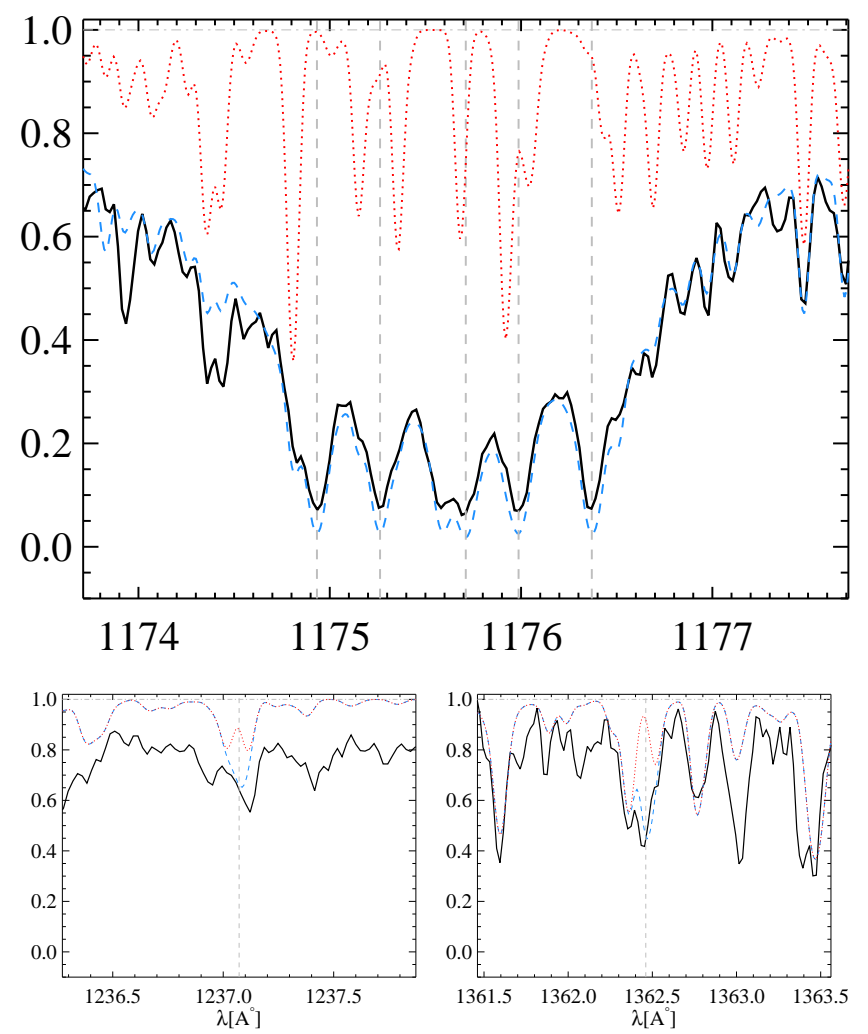

Figure 1. In this figure the $y$-axis represents the normalized flux and the $\mathrm{x}$-axis represents the wavelength in units of angstrom. The black curve is the observed spectrum of $\iota$-Herculis. The blue dashed line is the calculated model spectrum using the specified abundance and the red dotted line is the calculated model in the absence of that element. Top: The $C$ III lines of multiplet (4) around $1174 \AA$, with prominent wings extending to several $\AA$ from the deepest part of the feature. Bottom left:A strong line Ge II at

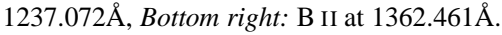

lines might be in the VALD database with incorrect $\log g f$ values. Using the "Extract all" option, we requested all lines in the database close to some of the strongest unidentified features, and examined the resulting short lists for good wavelength coincidence. We then considered how large a change in oscillator strength would be required to fit the observations, and whether such a change would be plausible. Although we found a few good wavelength coincidences, in all cases no reasonable change in oscillator strength would lead to a good fit. This line of investigation was eventually abandoned.

We suspect that most of the remaining strong unidentified features are high-excitation lines of abundant ions, perhaps usually of iron peak elements, that simply lack oscillator strengths.

\section{DISCUSSION AND CONCLUSIONS}

\subsection{Comparison with Literature}

This work presents a detailed UV spectral synthesis for Iota Herculis. It is important to compare the outcome of this new approach with previous work. In this project, we investigated the abundance of 27 elements starting from $Z=5$ up to $Z=80$. We could not determine the abundance of a few elements that we tried to detect, specifically neon $(Z=10)$, gold $(Z=79)$, and tin $(Z=50)$ because their spectral features were too weak and heavily blended to be useful for abundance analysis. Among the remaining 24 elements, there are 16 that have been studied in previous works. 


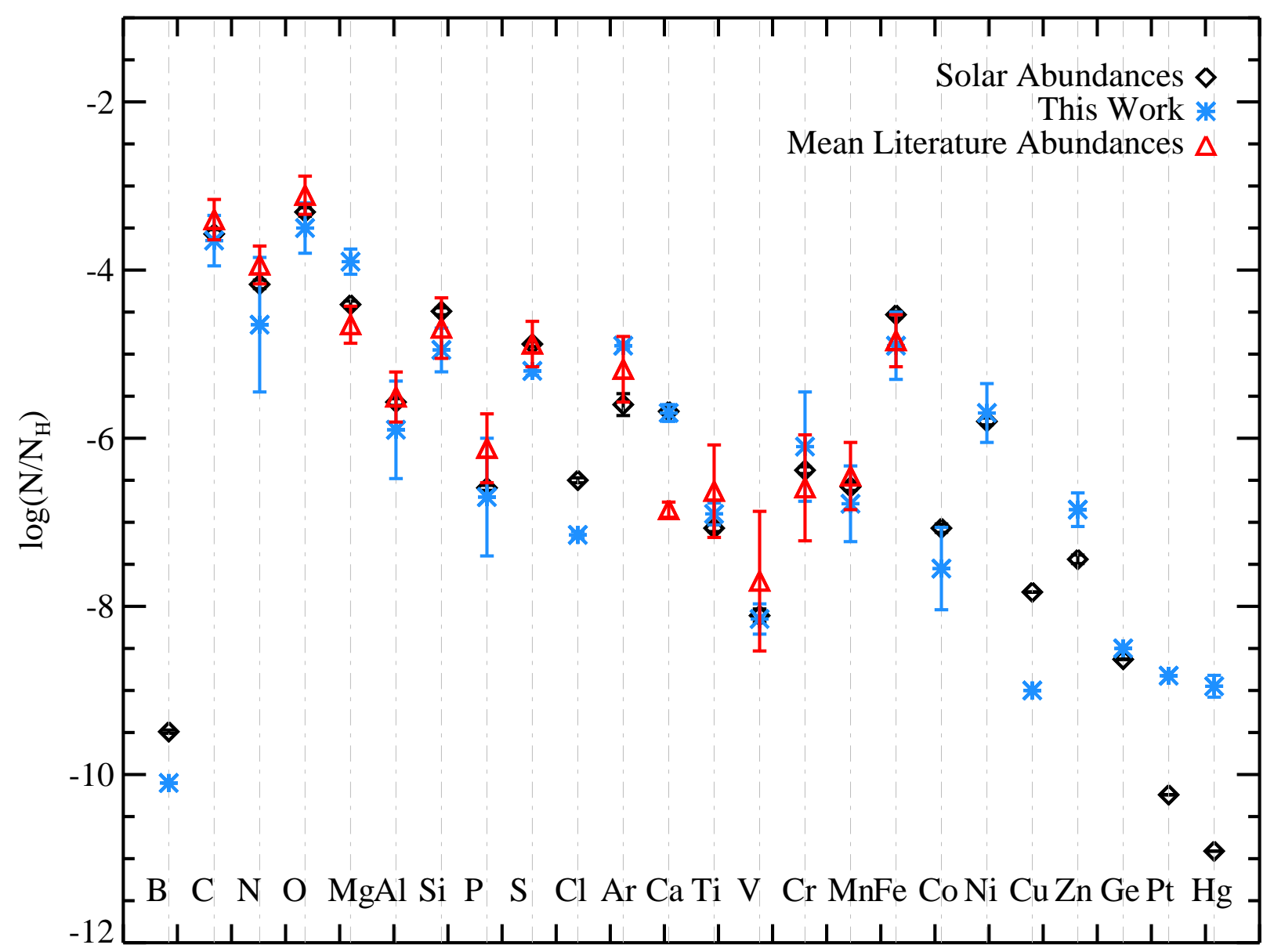

Figure 2. This figure shows a comparison between the solar abundances of 24 elements and the abundances derived from this work and the literature. Note that the literature values are the mean taken from Table 1

The results of this study are listed in Table 2 and shown graphically in Figure 2 All 16 elements, except for one, are in good agreement with previous work (see Table 1) within the estimated uncertainties. This indicates that the UV spectral synthesis is indeed a reasonable tool for abundance determination, at least for sharp-line stars with fundamental parameters similar to $\iota$ Her.

The only exception to this generalization is magnesium. We find a higher abundance of $-3.90 \pm 0.19$ for magnesium that even within the measured uncertainties, is still higher than previous estimates. This difference is probably due to the fact, discussed in Sec. 4.5, that the dominant ionization state for magnesium at this temperature is $\mathrm{Mg}$ III, but the line list contains only lines of $\mathrm{Mg}$ II. Thus abundance determinations for this element are likely to be affected by non-LTE effects which may well vary from line to line.

The line list used here does contain a few lines of gold $(Z=79)$, tin $(Z=50)$, and neon $(Z=10)$. These lines are found to be very weak and heavily blended. Thus, unfortunately, they do not enable us to determine the abundance of these elements. However, we have been able to estimate a value or an upper limit for the abundance of nine elements that were not previously studied, presumably because of a lack of useful lines in the optical wave- length window usually used for analysis; boron $(-10.10 \pm 0.10)$, chlorine (-7.15 \pm 0.05$)$, cobalt $(-7.55 \pm 0.49)$, nickel $(-5.70 \pm 0.36)$, copper (-9.00), zinc $(-6.85 \pm 0.24)$, germanium (-8.5), platinum ($8.825 \pm 0.100)$, and mercury $(-8.95 \pm 0.17)$. In the atmosphere of $\iota$ Herculis the abundance of boron is sub-solar, nickel, cobalt and germanium are at most almost solar, and platinum and mercury could even be higher than solar by 1-2 orders of magnitude.

Overall, the aims of this project, as described in $\$ 1$ have been fulfilled. In addition to identifying previously unreported elements, we find a good agreement with previous work. This conclusion probably only applies to quite sharp-line stars because of severe line blending even at very low $v \sin i$ values. We find that LTE modeling in the UV is a useful tool for determining elemental abundances, at least in stars with $T_{\text {eff }}$ not much higher than that of $\iota$ Her. Finally, in agreement with the results reported by Landstreet (2011), we find that the available VALD line lists seem to be missing a significant number of moderately strong lines (quite possibly many are high-excitation lines of iron peak elements), and that the incompleteness of the line lists appears to grow with decreasing wavelength, even over the limited wavelength range of this study. 


\subsection{Further Considerations and future work}

We have now carried out abundance analyses using using ultraviolet spectra analyzed with synthesis methods for three A or B stars: Sirius A $=$ HD 48915 from HST GHRS spectra of $R \approx 25000$ (Landstreet 2011), HD $72660=$ HR 3383 using STIS spectra of $R \approx 114000$ (Golriz \& Landstreet 2015), and $\iota$ Her using Copernicus spectra of $R \approx 24000$ or less (the present work). We have concluded that the technique of full spectrum synthesis of selected spectral windows makes it possible, in spite of the extreme line crowding in the UV in early-type stars, to carry out useful abundance analysis on the basis of such spectra. It is of interest to step back and look more broadly at what the limitations of this technique are for other spectral material and other stars.

We can first note an important feature of the available atomic data, as assembled by VALD. The line lists appear to become both less complete and to have less accurate atomic data as analysis moves father into the UV from the edge of the visible spectrum at about $3000 \AA$. This effect is strongly noticeable below about $1700 \AA$. In part this is a product of the limitations of the work done by the atomic physics community, but it also reflects the so far quite limited use of spectrum synthesis in the UV, resulting in line lists that have been far less carefully vetted for accuracy than those used in the visible window.

However, the accuracy of results also depends strongly on various characteristics of the stars studied and of the available spectra. The model spectra are more likely to be successful in fitting the observations for spectra of high S/N (say well above 100) and for high resolution (say 40000 or above so that individual lines are nearly or completely resolved), and for stars of very low $v \sin i$. The three stars analyzed by us all have $v \sin i$ of between 5 and $16 \mathrm{~km} / \mathrm{s}$. Thus IUE high-resolution spectra, obtained with resolving power of around $12-13000$ and $\mathrm{S} / \mathrm{N}$ of typically 30 or less, will necessarily be much more difficult to model with confidence. It will be very important to test line lists on suitable high-resolution UV spectra of sharp-line standard stars covering a range of effective temperature in order to empirically improve the quality of uncertain $g f$ values.

Improvement to the accuracy of much atomic data will certainly also be required if accurate determination of stellar parameters using ultraviolet spectra is to be attempted. The best determinations of basic parameters using visible spectra (e.g. Nieva \& Przybilla 2012) have been carried out on stars with sufficiently sparse line spectra that individual lines can easily be studied. This is simply not possible in the UV spectra of A and B stars of roughly solar abundance, and parameter determination will depend not only on accurate atomic data for selected lines, but also for the many possible blending lines.

We have searched our data for possible indications of a stellar wind. Numerous studies have shown that early type stars reveal the presence of a stellar wind primarily through blue-shifted excess absorption in the wings of resonance lines (Lamers 1981; Lamers et al. 1982). We have modeled resonance lines of C II at 1334-35 $\AA$, of N II at 1083-85 $\AA$, and of Si IV at 1393-1402 $\AA$. These appear to be the strongest such lines present in regions for which we have data and that we can model confidently (i.e. they are not in the wings of Lyman $\alpha$ or Lyman $\beta$. All these lines were modeled reasonably well (e.g. Figure 1), and in none of them did we find any hint of excess blue wing absorption. Modeling with a code able to model the Lyman lines, and/or data covering a larger wavelength window, could further clarify this situation. In any case, the absence of a detectable (mixed) stellar wind in this star is consistent with the wind computations of Babel (1996).
Diffusion is also another process that can occur in the stable atmosphere of slow rotating B stars. Under the action of gravity alone, heavier atoms would sink through the ambient hydrogen down into the stellar interior under the influence of gravity.

However, sometimes observation shows a large abundance of heavy elements (e.g. $\mathrm{Mn}, \mathrm{Sr}, \mathrm{Pt}, \mathrm{Hg}$, etc.) in the atmosphere, and an under-abundance of lighter elements (e.g. He, Ne, O, etc.). This kind of abundance peculiarity occurs when radiation pressure from the radiation flowing out of the stellar interior reverses the downward drift of low-abundance elements. This phenomenon is known as "radiative levitation". Radiative levitation can operate particularly effectively in slowly rotating stars, and although we do not know the actual rotation rate of $\iota$ Her, its very low $v \sin i$ value suggests that it may well be a slow rotator.

In the atmosphere of $\iota$ Herculis, we find,in agreement with other studies, that most of our light elements have almost solar abundances. For heavier elements such as zinc $(\mathrm{Z}=30)$, platinum $(Z=78)$ and mercury $(Z=80)$, our upper limits are unfortunately higher than the solar values. This is unfortunate, as it prevents us from looking for the phenomenon observed by Hill \& Landstreet (1993), that even most normal but slowly rotating A0V stars seem to have overabundance of some heavy elements such as $\mathrm{Zr}$, Ba and La.

Chemical abundances of stars provide probably the most powerful probes of their structure and of the complex mixing and separation processes that occur in the stellar interior and envelope and that influence evolution on the main sequence and in later stages. Obtaining accurate measurements of the relative abundances of as many elements as possible is an essential first step to interpreting the information content of stellar chemistry. This work has shown that the ultraviolet wavelength window can make a very useful contribution to this step towards a better understanding of such sharpline mid-B stars as $\iota$ Her.

\section{ACKNOWLEDGMENTS}

SSG acknowledges support from the Department of Physics and Astronomy of the University of Western Ontario. SSG and JDL acknowledge financial support for this work from the Natural Sciences and Engineering Council of Canada. This work is based on observations made with Copernicus satellite or Orbiting Astronomical Observatory 3 (OAO-3), a collaborative effort between the USA(NASA) and the UK(SERC). The UV telescope on board belonged to the Princeton University. Some of the data presented in this paper were obtained from the Mikulski Archive for Space Telescopes (MAST). STScI is operated by the Association of Universities for Research in Astronomy, Inc., under NASA contract NAS5-26555. Support for MAST for non-HST data is provided by the NASA Office of Space Science via grant NNX13AC07G and by other grants and contracts. 
Table 1. Iota Herculis- Summary of literature abundances. (1) Pintado \& Adelman (1993); (2) Schmidt (1979); (3) Balona (1984); (4) Lester et al. (1986); (5) Kane et al. (1980); (6) Barnett \& McKeith (1988); (7) Grigsby (1991); (8) Dufton et al. (1981); (9) Peters \& Polidan (1985) ; (10) Peters \& Aller (1970); (11) Nieva \& Przybilla (2012). Note: The first eleven columns represent abundances from difference sources and the last column represents the solar abundances which are taken from Scott et al. (2014, I \& II), Grevesse et al. (2014) and (Asplund et al. 2009)

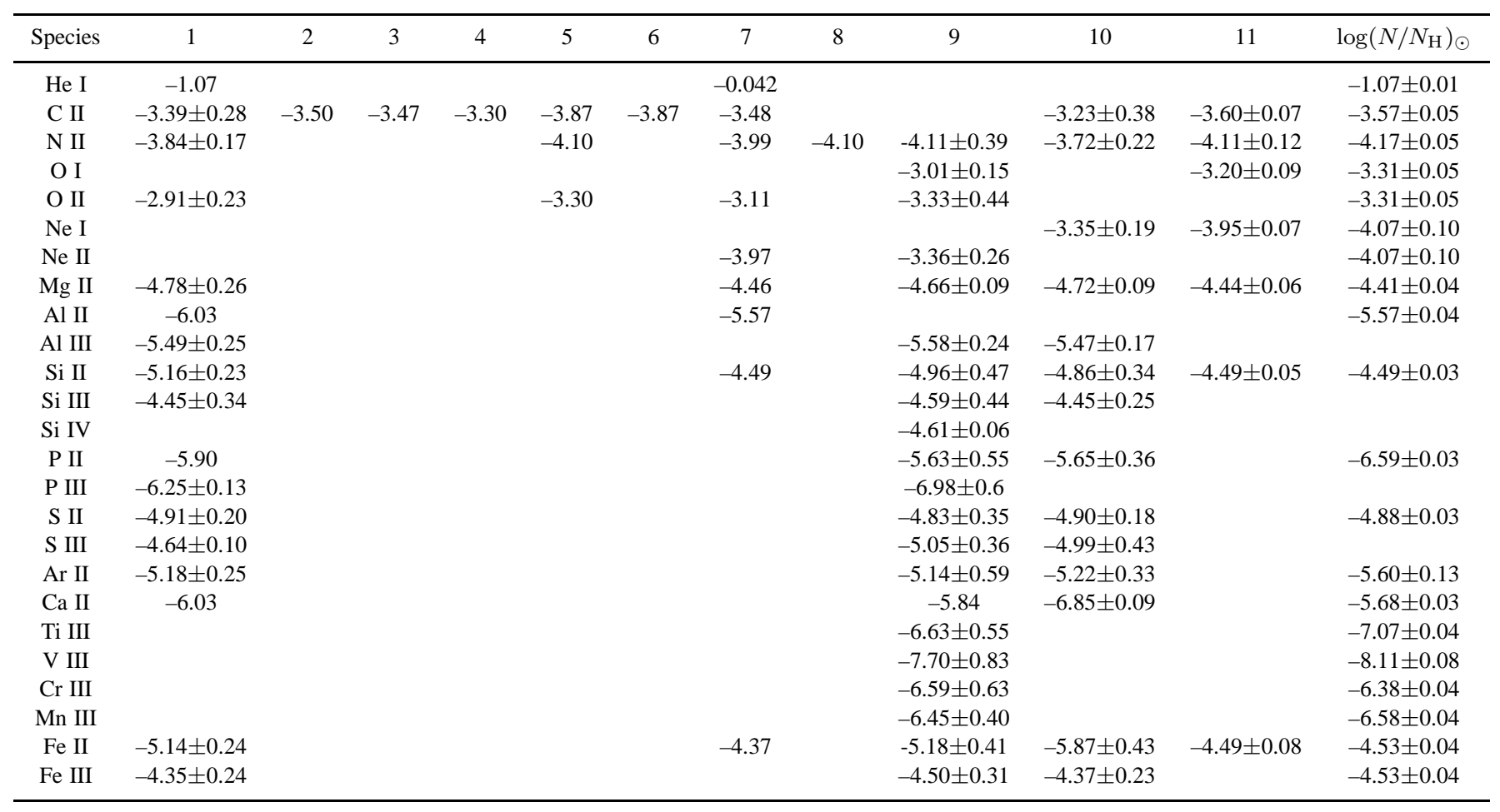


Table 2. This table shows the abundances determined in this work for every ion and the recommended value for every element, the solar abundances from Scott et al. (2014, I \& II), Grevesse et al. (2014) and Asplund et al. (2009), and the wavelengths used to determine these values. Note that the recommended value is the variance of all the available literature values. The subset of VALD list used in our models can be found here: www.astro.uwo.ca/ jlandstr/iota_her_linelist/linelist.txt

\begin{tabular}{|c|c|c|c|}
\hline $\begin{array}{l}\text { Element } \\
\text { or ion }\end{array}$ & $\begin{array}{l}\text { Abundance } \\
\text { (this work) }\end{array}$ & $\log \left(N / N_{\mathrm{H}}\right)$ & $\log \left(N / N_{\mathrm{H}}\right)_{\odot}$ \\
\hline B II & $-10.100 \pm 0.100$ & $-10.100 \pm 0.100$ & $-9.300 \pm 0.020$ \\
\hline $\mathrm{C}_{\text {II }}$ & & $-4.450 \pm 0.300$ & \\
\hline C III & & $-3.750 \pm 0.250$ & \\
\hline $\mathrm{C}$ & $-3.55 \pm 0.300$ & & $-3.570 \pm 0.05$ \\
\hline $\mathrm{N}_{\mathrm{I}}$ & & $-4.350 \pm 0.340$ & \\
\hline N II & & $-4.860 \pm 0.340$ & \\
\hline $\mathrm{N}$ & $-4.650 \pm 0.800$ & & $-4.170 \pm 0.050$ \\
\hline O I & & $-3.500 \pm 0.300$ & \\
\hline O II & & $-3.550 \pm 0.300$ & \\
\hline $\mathrm{O}$ & $-3.500 \pm 0.300$ & & $-3.310 \pm 0.050$ \\
\hline Mg II & $-3.900 \pm 0.150$ & $-3.900 \pm 0.190$ & $-4.410 \pm 0.040$ \\
\hline $\mathrm{Al}$ III & $-5.900 \pm 0.580$ & $-5.900 \pm 0.580$ & $-5.570 \pm 0.040$ \\
\hline Si II & & $-4.650 \pm 0.27$ & \\
\hline Si III & & $-4.950 \pm 0.20$ & \\
\hline Si IV & & $-4.750 \pm 0.15$ & \\
\hline $\mathrm{Si}$ & $-4.780 \pm 0.260$ & & $-4.490 \pm 0.030$ \\
\hline P III & & $-6.750 \pm 0.680$ & \\
\hline$P_{\text {II }}$ & & $-6.400 \pm 0.660$ & \\
\hline $\mathrm{P}$ & $-6.700 \pm 0.700$ & & $-6.590 \pm 0.030$ \\
\hline S II & & $-5.300 \pm 0.050$ & \\
\hline S III & & $-5.200 \pm 0.100$ & \\
\hline S IV & & $-5.200 \pm 0.100$ & \\
\hline S & $-5.200 \pm 0.100$ & & $-4.880 \pm 0.030$ \\
\hline $\mathrm{Cl}$ II & $-7.150 \pm 0.100$ & $-7.150 \pm 0.041$ & $-6.500 \pm 0.030$ \\
\hline ArI & $-4.900 \pm 0.100$ & $-4.900 \pm 0.041$ & $-5.600 \pm 0.13$ \\
\hline Ca II & $-5.700 \pm 0.100$ & $-5.700 \pm 0.010$ & $-5.680 \pm 0.030$ \\
\hline Ti III & $-6.900 \pm 0.170$ & $-6.900 \pm 0.130$ & $-7.070 \pm 0.040$ \\
\hline V III & $-8.150 \pm 0.180$ & $-8.150 \pm 0.180$ & $-8.110 \pm 0.080$ \\
\hline Cr III & $-6.100 \pm 0.650$ & $-6.100 \pm 0.650$ & $-6.380 \pm 0.040$ \\
\hline Mn III & $-6.780 \pm 0.450$ & $-6.780 \pm 0.450$ & $-6.580 \pm 0.040$ \\
\hline Fe II & & $-4.950 \pm 0.390$ & \\
\hline $\mathrm{Fe}$ III & & $-4.750 \pm 0.400$ & \\
\hline $\mathrm{Fe}$ & $-4.900 \pm 0.400$ & & $-4.530 \pm 0.040$ \\
\hline Co II & & $-7.550 \pm 0.100$ & \\
\hline Co III & & $-7.450 \pm 0.490$ & \\
\hline Co & $-7.55 \pm 0.490$ & & $-7.070 \pm 0.050$ \\
\hline Ni II & & $-5.500 \pm 0.360$ & \\
\hline Ni III & & $-5.800 \pm 0.300$ & \\
\hline $\mathrm{Ni}$ & $-5.700 \pm 0.350$ & & $-5.800 \pm 0.040$ \\
\hline $\mathrm{Cu}$ II & -9.000 & & -7.830 \\
\hline Zn III & $-6.850 \pm 0.200$ & $-6.850 \pm 0.200$ & $-7.440 \pm 0.050$ \\
\hline Ge II & -8.500 & & -8.630 \\
\hline Pt III & -8.825 & -8.825 & -10.240 \\
\hline $\mathrm{Hg}$ III & $-8.950 \pm 0.130$ & $-8.950 \pm 0.130$ & -10.91 \\
\hline
\end{tabular}


Table 3. This table contains a list of adopted atomic data for the most useful and important spectral lines used in the modelling of various chemical elements in the UV spectrum of $\iota$ Her.

\begin{tabular}{|c|c|c|c|c|}
\hline Spec Ion & $\begin{array}{c}W L_{\mathrm{vac}} \\
\text { (A) }\end{array}$ & $\log g f$ & $\begin{array}{c}E_{\text {low }} \\
(\mathrm{eV})\end{array}$ & Ref \\
\hline 'B 2' & 1362.4610 & -0.076 & 0.000 & VALD \\
\hline 'C 3' & 1174.9330 & -0.468 & 6.496 & VALD \\
\hline 'C 3' & 1175.2630 & -0.565 & 6.493 & VALD \\
\hline 'C 3' & 1175.5900 & -0.690 & 6.496 & VALD \\
\hline 'C 3' & 1175.7110 & 0.009 & 6.503 & VALD \\
\hline 'C 3' & 1175.9870 & -0.565 & 6.496 & VALD \\
\hline 'C 3' & 1176.3700 & -0.468 & 6.503 & VALD \\
\hline 'C 3' & 1247.3830 & -0.314 & 12.690 & VALD \\
\hline 'C 2' & 1323.8617 & -1.284 & 9.290 & VALD \\
\hline 'C 2' & 1323.9059 & -0.337 & 9.291 & VALD \\
\hline 'C 2' & 1323.9510 & -0.144 & 9.290 & VALD \\
\hline 'C 2', & 1323.9950 & -1.288 & 9.291 & VALD \\
\hline 'C 1' & 1328.8333 & -1.236 & 0.000 & VALD \\
\hline 'C 1' & 1329.0849 & -1.231 & 0.002 & VALD \\
\hline 'C 1' & 1329.1000 & -1.147 & 0.002 & VALD \\
\hline 'C 1' & 1329.1230 & -1.355 & 0.002 & VALD \\
\hline 'C 1' & 1329.5775 & -0.662 & 0.005 & VALD \\
\hline 'C 1' & 1329.6000 & -1.136 & 0.005 & VALD \\
\hline 'C 2' & 1334.5320 & -0.589 & 0.000 & VALD \\
\hline 'C 2' & 1335.6627 & -1.293 & 0.008 & VALD \\
\hline 'C 2' & 1335.7077 & -0.335 & 0.008 & VALD \\
\hline 'N 2' & 1085.5290 & -2.277 & 0.016 & VALD \\
\hline 'N 2' & 1085.5460 & -1.095 & 0.016 & VALD \\
\hline 'N 2' & 1085.7010 & -0.337 & 0.016 & VALD \\
\hline 'N 1' & 1199.5500 & -0.278 & 0.000 & VALD \\
\hline 'N 1' & 1200.2230 & -0.459 & 0.000 & VALD \\
\hline 'N 1' & 1200.7100 & -0.762 & 0.000 & VALD \\
\hline 'N 1' & 1243.1710 & -1.511 & 2.384 & VALD \\
\hline 'N 1' & 1243.1790 & -0.349 & 2.384 & VALD \\
\hline 'N 1' & 1243.3060 & -0.541 & 2.385 & VALD \\
\hline 'N 1' & 1243.3130 & -1.484 & 2.385 & VALD \\
\hline 'N 2' & 1275.0380 & -1.206 & 11.436 & VALD \\
\hline 'N 2' & 1275.2510 & -1.944 & 11.438 & VALD \\
\hline 'N 2', & 1276.2010 & -1.478 & 11.438 & VALD \\
\hline 'N 2' & 1276.2250 & -1.948 & 11.438 & VALD \\
\hline 'N 2' & 1276.8000 & -1.828 & 11.438 & VALD \\
\hline 'O 1' & 1039.2300 & -1.339 & 0.000 & VALD \\
\hline 'O 1' & 1040.9430 & -1.561 & 0.020 & VALD \\
\hline 'O 1' & 1041.6880 & -2.037 & 0.028 & VALD \\
\hline 'O 2' & 1132.3890 & -1.854 & 14.889 & VALD \\
\hline 'O 1' & 1302.1680 & -0.585 & 0.000 & VALD \\
\hline 'O 1' & 1304.8580 & -0.808 & 0.020 & VALD \\
\hline 'O 1', & 1306.0290 & -1.285 & 0.028 & VALD \\
\hline 'Mg 2' & 1239.9250 & -3.530 & 0.000 & VALD \\
\hline 'Mg 2' & 1240.3950 & -3.830 & 0.000 & VALD \\
\hline 'Mg 2' & 1367.2570 & -2.080 & 4.434 & VALD \\
\hline 'Mg 2' & 1367.7080 & -2.240 & 4.422 & VALD \\
\hline 'Al 2' & 1190.0460 & -1.260 & 4.644 & VALD \\
\hline 'Al 2' & 1190.0510 & -0.780 & 4.644 & VALD \\
\hline 'Al 2' & 1191.8080 & -1.260 & 4.659 & VALD \\
\hline 'Al 2' & 1191.8140 & -0.510 & 4.659 & VALD \\
\hline 'Al 3' & 1352.8100 & -0.020 & 14.377 & VALD \\
\hline 'Al 3' & 1352.8580 & -0.180 & 14.377 & VALD \\
\hline 'Al 3' & 1379.6700 & -0.600 & 6.656 & VALD \\
\hline 'Si 4' & 1128.3250 & -0.480 & 8.896 & VALD \\
\hline 'Si 4' & 1128.3400 & 0.470 & 8.896 & VALD \\
\hline 'Si 2' & 1190.4158 & -0.245 & 0.000 & VALD \\
\hline
\end{tabular}


Table 4. The percentage ionization ratios derived from the Saha equation. Here $n_{t o t}$ is the total number density in every state $\left(n_{t o t}=n_{0}+n_{1}+n_{2}+n_{3}\right)$. This table shows the ionization balances in two different regions; left column: the region where the continuum and weak lines are formed $\left(\tau_{5000} \sim 0.7\right.$, $\tau_{1110} \sim \tau_{1320} \sim 0.2$, where $\left.T \approx 17000 \mathrm{~K}, n_{e} \approx 3.8410^{14}\right)$ and right column: the region where line cores of strong lines are formed $\left(\tau_{5000} \sim \tau_{1110} \sim\right.$ $\tau_{1320} \sim 710^{-4}$, where $T \approx 11800 \mathrm{~K}, n_{e} \approx 5.1310^{12}$ ) respectively (Kurucz 1979 ).

\begin{tabular}{|c|c|c|c|c|c|c|c|c|}
\hline Species & {$[\mathrm{I}] / n_{t o t}$} & $\begin{array}{r}\tau=0.2 \\
{[\mathrm{II}] / n_{\text {tot }}}\end{array}$ & {$[\mathrm{III}] / n_{\text {tot }}$} & {$[\mathrm{IV}] / n_{t o t}$} & {$[\mathrm{I}] / n_{\text {tot }}$} & $\begin{array}{l}\tau=7 \times 10^{-4} \\
{[\mathrm{II}] / n_{\text {tot }}}\end{array}$ & {$[\mathrm{III}] / n_{\text {tot }}$} & {$[\mathrm{IV}] / n_{t o t}$} \\
\hline B & 0.002 & 27.30 & 72.69 & 0.009 & $2.07 \times 10^{-3}$ & 97.52 & 2.50 & $4.25 \times 10^{-8}$ \\
\hline $\mathrm{C}$ & 0.007 & 72.84 & 27.14 & $1.71 \times 10^{-5}$ & 0.01 & 99.53 & 0.46 & 0.00 \\
\hline $\mathrm{N}$ & 0.03 & 95.68 & 4.29 & $3.03 \times 10^{-7}$ & 0.08 & 99.91 & $9.76 \times 10^{-3}$ & 0.00 \\
\hline $\mathrm{O}$ & 0.073 & 99.55 & 0.37 & 0.00 & 0.17 & 99.83 & $1.3 \times 10^{-4}$ & 0.00 \\
\hline $\mathrm{Ne}$ & 1.11 & 98.88 & 0.005 & 0.00 & 26.31 & 73.70 & $1.81 \times 10^{-7}$ & 0.00 \\
\hline $\mathrm{Mg}$ & $4.98 \times 10^{-7}$ & 0.17 & 99.83 & 0.00 & $5.60 \times 10^{-7}$ & 0.60 & 99.40 & 0.00 \\
\hline $\mathrm{Al}$ & $5.80 \times 10^{-6}$ & 0.49 & 92.73 & 6.77 & $1.34 \times 10^{-5}$ & 6.42 & 93.56 & $2.20 \times 10^{-2}$ \\
\hline $\mathrm{Si}$ & $1.49 \times 10^{-5}$ & 1.19 & 97.84 & 0.97 & $2.93 \times 10^{-5}$ & 6.39 & 93.61 & $5.53 \times 10^{-4}$ \\
\hline$P$ & $5.02 \times 10^{-5}$ & 2.84 & 96.42 & 0.73 & $4.75 \times 10^{-4}$ & 33.66 & 66.34 & $9.06 \times 10^{-4}$ \\
\hline$S$ & $7.30 \times 10^{-4}$ & 8.92 & 90.96 & 0.12 & $5.35 \times 10^{-3}$ & 84.85 & 15.15 & $7.78 \times 10^{-6}$ \\
\hline $\mathrm{Cl}$ & $5.70 \times 10^{-3}$ & 40.64 & 59.34 & 0.01 & $2.50 \times 10^{-2}$ & 97.84 & 2.13 & $3.05 \times 10^{-8}$ \\
\hline $\mathrm{Ar}$ & 0.02 & 72.46 & 27.52 & $4.00 \times 10^{-4}$ & 0.11 & 99.74 & 0.16 & 0.00 \\
\hline $\mathrm{Ca}$ & $2.13 \times 10^{-8}$ & 0.02 & 99.98 & $2.48 \times 10^{-5}$ & 0.00 & $2.55 \times 10^{-2}$ & 99.97 & 0.00 \\
\hline $\mathrm{Ti}$ & $9.53 \times 10^{-8}$ & 0.04 & 88.28 & 11.68 & $5.68 \times 10^{-8}$ & $9.44 \times 10^{-2}$ & 99.85 & $5.80 \times 10^{-2}$ \\
\hline V & $1.98 \times 10^{-7}$ & 0.05 & 94.16 & 5.78 & $1.53 \times 10^{-7}$ & 0.18 & 99.80 & $1.47 \times 10^{-2}$ \\
\hline $\mathrm{Cr}$ & $2.01 \times 10^{-7}$ & 0.05 & 97.01 & 2.94 & $2.82 \times 10^{-7}$ & 0.32 & 99.68 & $4.20 \times 10^{-3}$ \\
\hline $\mathrm{Mn}$ & $6.37 \times 10^{-7}$ & 0.14 & 98.06 & 1.79 & $8.30 \times 10^{-7}$ & 0.65 & 99.35 & $1.02 \times 10^{-3}$ \\
\hline $\mathrm{Fe}$ & $1.27 \times 10^{-6}$ & 0.22 & 98.99 & 0.79 & $2.30 \times 10^{-6}$ & 1.15 & 98.85 & $1.21 \times 10^{-3}$ \\
\hline $\mathrm{Co}$ & $2.23 \times 10^{-6}$ & 0.24 & 99.32 & 0.43 & $5.22 \times 10^{-6}$ & 1.72 & 98.28 & $2.60 \times 10^{-4}$ \\
\hline $\mathrm{Ni}$ & $4.03 \times 10^{-6}$ & 0.33 & 99.46 & 0.21 & $1.25 \times 10^{-5}$ & 3.27 & 96.73 & $7.07 \times 10^{-5}$ \\
\hline $\mathrm{Cu}$ & $3.57 \times 10^{-6}$ & 0.29 & 99.60 & 0.11 & $2.25 \times 10^{-5}$ & 5.64 & 94.36 & $2.05 \times 10^{-5}$ \\
\hline $\mathrm{Zn}$ & $1.14 \times 10^{-5}$ & 1.19 & 98.73 & 0.07 & $5.50 \times 10^{-5}$ & 10.36 & 89.64 & $5.10 \times 10^{-6}$ \\
\hline $\mathrm{Ge}$ & $9.60 \times 10^{-6}$ & 0.91 & 98.49 & 0.60 & $1.54 \times 10^{-5}$ & 4.32 & 95.67 & $2.72 \times 10^{-4}$ \\
\hline Sn & $2.60 \times 10^{-6}$ & 0.36 & 92.80 & 6.84 & $2.46 \times 10^{-6}$ & 1.21 & 98.78 & $1.17 \times 10^{-2}$ \\
\hline $\mathrm{Pt}$ & $1.07 \times 10^{-5}$ & 0.75 & 82.89 & 16.36 & $6.54 \times 10^{-5}$ & 9.53 & 90.41 & $6.61 \times 10^{-2}$ \\
\hline $\mathrm{Au}$ & $5.20 \times 10^{-5}$ & 3.04 & 92.20 & 4.75 & $3.81 \times 10^{-4}$ & 42.40 & 57.60 & $5.65 \times 10^{-3}$ \\
\hline $\mathrm{Hg}$ & $1.95 \times 10^{-5}$ & 1.01 & 98.68 & 0.30 & $1.72 \times 10^{-4}$ & 11.34 & 88.66 & $1.30 \times 10^{-4}$ \\
\hline
\end{tabular}




\section{REFERENCES}

Asplund, M., Grevesse, N., Sauval, A. J., \& Scott, P. 2009, ARA\&A, 47, 481

Aerts, C., Puls, J., Godart, M., \& Dupret, M.-A. 2009, Communications in Asteroseismology, 158, 66

Biémont, E., Palmeri, P., \& Quinet, P. 1999, Ap\&SS , 269, 635

Babel, J. 1996, A\&A , 309, 867

Bailey, J. D., \& Landstreet, J. D. 2013, A\&A, 551, AA30

Balona, L. A. 1984, MNRAS, 211, 973

Barnett, E. W., \& McKeith, C. D. 1988, MNRAS, 234, 325

Cantiello, M., Langer, N., Brott, I., et al. 2009, Communications in Asteroseismology, 158, 61

Chapellier, E., Mathias, P., Le Contel, J.-M. et al. 2000, A\&A, 362, 189

Cowley, C. R. 1996, M.A.S.S., Model Atmospheres and Spectrum Synthesis, 108, 223

Dufton, P. L., \& McKeith, C. D. 1980, $A \& A, 81,8$

Dufton, P. L., Kane, L., \& McKeith, C. D. 1981, MNRAS, 194, 85

Golriz, S. S., Landstreet, J. D. 2015 , MNRAS, 456, 3

Grigsby, J. A. 1991, ApJ, 380, 606

Grigsby, J. A., Mulliss, C. L., \& Baer, G. M. 1996, PASP, 108, 953

Hill, G. M., \& Landstreet, J. D. 1993, A\&A, 276, 142

Kramida, A., Ralchenko, Yu., Reader, J. and NIST ASD Team, 2014, NIST Atomic Spectra Database (version 5.2), [Online]. Available: http://physics.nist.gov/asd [Saturday, 24-Jan2015 16:37:16 EST]. National Institute of Standards and Technology, Gaithersburg, MD

Klinglesmith, D. A., III, Sonneborn, G., Polidan, R. S., et al. 1996, Astronomical Data Analysis Software and Systems V, 101, 521

Kane, L., McKeith, C. D., \& Dufton, P. L. 1980, A\&A, 84, 115

Kupka, F., Piskunov, N., Ryabchikova, T. A., Stempels, H. C., \& Weiss, W. W. 1999, A\&AS, 138, 119

Kupka, F. G., Ryabchikova, T. A., Piskunov, N. E., Stempels, H. C., \& Weiss, W. W. 2000, Baltic Astronomy, 9, 590

Kurucz, R. L. 1979, ApJS, 40, 1

Kurucz, R. L. 1970, SAO Special Report, 309,

Lanz, T., \& Hubeny, I. 2007, ApJS , 169, 83

Landsman, W. B. 1993, Astronomical Data Analysis Software and Systems II, 52, 246

Lamers, H. J. G. L. M. 1981, IAU Colloq. 59: Effects of Mass Loss on Stellar Evolution, 89, 181

Lamers, H. J. G. L. M., Gathier, R., \& Snow, T. P., Jr. 1982, ApJ, 258,186

Landstreet, J. D. 1988, ApJ, 326, 967

Landstreet, J. D., Barker, P. K., Bohlender, D. A., \& Jewison, M. S. $1989, A p J, 344,876$

Lennon, D. J. 1983, MNRAS, 205, 829

Lester, J. B., Gray, R. O., \& Kurucz, R. L. 1986, ApJS, 61, 509

Landstreet, J. D. 2011, A\&A, 528, AA132

Nieva, M.-F., \& Simón-Díaz, S. 2011, A\&A, 532, AA2

Nieva, M.-F., \& Przybilla, N. 2012, A\&A , 539, A143

Peters, G. J., \& Aller, L. H. 1970, ApJ, 159, 525

Peters, G. J., \& Polidan, R. S. 1985, Calibration of Fundamental Stellar Quantities, 111, 417

Pintado, O. I., \& Adelman, S. J. 1993, MNRAS, 264, 63

Przybilla, N., Nieva, M.-F., \& Butler, K. 2011, Journal of Physics Conference Series, 328, 012015

Piskunov, N., \& Kupka, F. 2001, ApJ, 547, 1040

Ross, C., B. Jr., Dissertation, Purdue Univ., Purdue, IN (1969)

Ryabtsev, A., N., Kink, I., Awaya, Y., Ekberg, J., O, Mannervik,
S. , lme, A. , \& Martinson, I., Phys. Scr. 71, 489501 (2005) DOI:10.1238/Physica.Regular.071a00489

Ryabchikova, T. A., Hill, G. M., Landstreet, J. D., Piskunov, N., \& Sigut, T. A. A. 1994, MNRAS , 267, 697

Rogerson, J. B., Spitzer, L., Drake, J. F., et al. 1973, ApJ , 181, L97

Rogerson, J. B., Jr., \& Upson, W. L., II 1977, ApJS , 35, 37

Ross, J. E., \& Aller, L. H. 1976, Science, 191, 1223

Ryabchikova, T. A., Piskunov, N. E., Kupka, F., \& Weiss, W. W. 1997, Baltic Astronomy, 6, 244

Moore, C., E., 1970, Nat. Stand. Ref. Data Ser., NSRDS-NBS 3 (Sect. 3), 73 pp. (Nat. Bur. Stand., U.S., 1970)

Swamy, K. S. K., \& Tarafdar, S. P. 1972, A\&A, 18, 415

Grevesse, N., Scott, P., Asplund, M., \& Sauval, A. J. 2014, arXiv: 1405.0288

Scott, P., Asplund, M., Grevesse, N., Bergemann, M., \& Sauval, A. J. 2014, arXiv: 1405.0287

Scott, P., Grevesse, N., Asplund, M., et al. 2014, arXiv:1405.0279

Spitzer, L., Jr., \& Morton, W. A. 1976, ApJ , 204, 731

Spitzer, L. 1976, QJRAS , 17, 97

Simón-Díaz, S., \& Herrero, A. 2014, A\&A, 562, A135

Schmidt, E. G. 1979, AJ, 84, 1739

Tachiev, G., \& Froese Fischer, C., J. Phys. B 33, 24192435 (2000) DOI:10.1088/0953-4075/33/13/304

Piskunov, N. E., Kupka, F., Ryabchikova, T. A., Weiss, W. W., \& Jeffery, C. S. 1995, A\&AS, 112, 525

Upson, W. L., II, \& Rogerson, J. B., Jr. 1980, ApJS, 42, 175

Vangioni-Flam, E., Cassé, M., \& Audouze, J. 2000, Phys. Rep. , 333, 365

Wade, G. A., Bagnulo, S., Kochukhov, O., et al. 2001, $A \& A$, 374, 265

Wade, G. A., Folsom, C. P., Petit, P., et al. 2014, arXiv:1407.3991

This paper has been typeset from a $\mathrm{T}_{\mathrm{E}} \mathrm{X} / \mathrm{LT}_{\mathrm{E}} \mathrm{X}$ file prepared by the author. 